\title{
REVIEW
}

\section{From individual to collective chirality in metal nanoparticles}

\author{
Andrés Guerrero-Martínez a,*, José Lorenzo Alonso-Gómez ${ }^{\mathrm{b}}$, \\ Baptiste Auguiéa ${ }^{\mathrm{a}}$ M. Magdalena Cid ${ }^{\mathrm{b}}$, Luis M. Liz-Marzán ${ }^{\mathrm{a}, *}$
}

a Colloid Chemistry Group, Departamento de Química Física and Unidad Asociada CSIC-Universidade de Vigo, 36310 Vigo, Spain

b Departamento de Química Orgánica, Universidade de Vigo, 36310 Vigo, Spain

Received 23 March 2011; received in revised form 7 June 2011; accepted 19 June 2011

Available online 22 July 2011

\author{
KEYWORDS \\ Chirality; \\ Plasmonics; \\ Metal nanoparticles; \\ Circular dichroism; \\ Optical activity; \\ Coupled-dipole model
}

\begin{abstract}
Recent reports have illustrated the promising potential of chiral metal nanostructures, which exploit the characteristic localized surface plasmon resonance of metal colloids, to produce intense optical activity. In this article we review the concepts, synthetic methods, and theoretical predictions underlying the chirality of metal colloids with a particular emphasis on the size range of 10-100 nanometers. The formation of individual colloidal nanoparticles with a chiral morphology and a plasmonic response remains elusive; however, collective chirality and the associated optical activity in nanoparticle assemblies is a promising alternative that has seen a few recent experimental demonstrations. We conclude with a perspective on chiral nanostructures built up from achiral anisotropic metal particles.
\end{abstract}

(c) 2011 Elsevier Ltd. All rights reserved.

\section{Introduction}

The term chirality takes us back to the 19th and 20th centuries, witnessing a great scientific effort dedicated to the investigation of the intimate structure-property relationships of matter, well beyond its macroscopic aspect. This interest has continued rising ever since and is, in fact, one of the cornerstones of today's fundamental and applied research. The advances and availability of increasingly powerful structural elucidation techniques have conducted

\footnotetext{
* Corresponding authors. Tel.: +34 986812298;

fax: +349868122556 .

E-mail addresses: aguerrero@uvigo.es (A. Guerrero-Martínez), Lmarzan@uvigo.es (L.M. Liz-Marzán).
}

the discovery of chemical and biological architectures, among which dissymmetric systems - also termed chiral often play a fundamental role. The realization that Nature assumes a ubiquitous relationship between structure and function has perhaps never been as clear as with chiral systems (Fig. 1) [1]. Following Pasteur's work on the resolution of tartaric acid [2], the development of efficient chemical syntheses of chiral species has been a driving force in the broad field of chemistry due to the important applications that such systems can find in quantitative analysis [3], catalysis [4], and pharmacology [5], among many others. As molecular biology reached a prominent role in the germination of new scientific ideas, three-dimensional (3D) molecular models were eagerly demanded to explain the specific handedness of biomacromolecules [6]. In this context, the successful investigation of Watson and Crick 


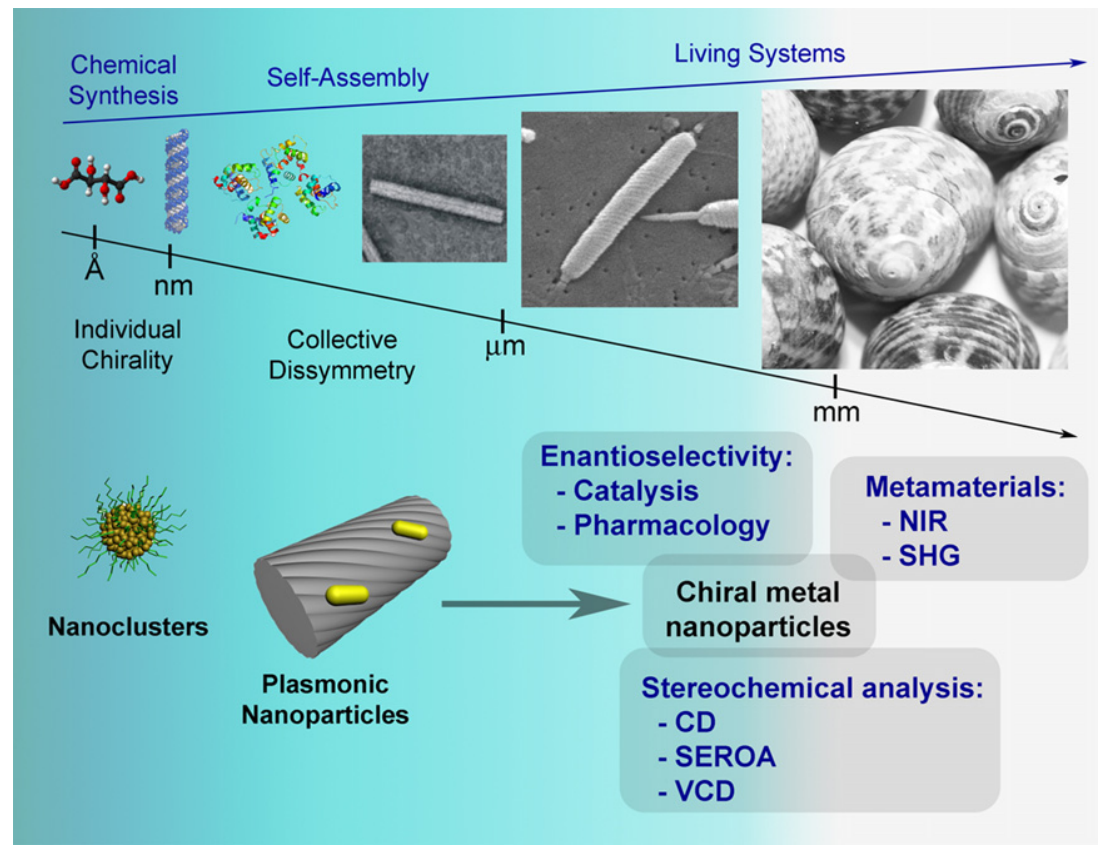

Figure 1 Classification of chiral architectures in Nature, from enantiomeric molecules at the Ångström scale (tartaric acid), to nano-sized biomacromolecules with chiral structures (DNA and proteins), microorganisms (helix-shaped viruses and bacteria such as Tobacco mosaic virus and Helicobacter pylori, respectively), and macroscopic living systems (snails like Helix pomatia). The complexity increases along the arrows with the cooperation of chiral subunits. Analogously, chirality in metal nanoparticles can be reached stepwise from individual nanoclusters and nanoparticles to directed-assembly of several plasmonic nanoparticles into 3D chiral structures. Potential applications in the preparation of metamaterials with photonic properties (Second Harmonic Generation (SHG), Negative Index of Refraction (NIR)); enantioselectivity in catalysis and pharmacology; and in stereochemical analysis by circular dichroism (CD), vibrational circular dichroism (VCD), and surface enhanced Raman optical activity (SEROA) are highlighted.

outlining the double-helix structure of DNA [7] is one outstanding example that broadened our knowledge about the conditions governing vital functions. Indeed, microorganisms such as viruses and bacteria synthesize relatively small chiral components (e.g., proteins) and assemble them through cooperative interactions to produce superstructures with replicated dissymmetry, which may be expressed in their external morphology at the micron scale [8]. Further, in an extension of this chiral expression, many macroscopic living systems are composed of body segments with twisted or helical shapes at the mesoscale, which seem to have optical evolutionary functions [9].

Concurrently to the study of naturally occurring substances, the elaboration of artificial systems with controlled material properties has given rise to a rich set of fabrication techniques that allow a fine control over the morphology down to the nanoscale, though it often lacks the apparent effortlessness with which Nature has achieved complex structures, chiral in particular (Fig. 1) [10]. The interdisciplinary character of nanoscience and nanotechnology [11], in which chemical and biological entities may be used as key ingredients in the construction of larger systems through a bottom-up approach, provides an excellent playground to explore the preparation of new dissymmetric materials at the nanoscale. Remarkable examples of materials that are widely used in nanotechnology are mesoporous silica [12], carbon nanotubes [13], quantum dots [14], and metal nanoparticles [15].
A striking example of artificial structures that reach beyond naturally occurring substances is the emerging field of metamaterials [16], concerning in particular nanostructures displaying novel optical properties. Here again, chiral elements have been sought as promising candidates for exotic optical functions as in non-linear optics [17], or negative refraction $[18,19]$. More generally, many applications of nanoscale chirality can benefit from optical characterization; chiral nanostructured systems in particular are currently being investigated for their use as powerful probes upon interaction with chiral biomacromolecules (e.g., proteins) [20].

Coinciding with early studies on chirality, but until recently following a completely different path, the study of colloidal metal particles traces back to the 19th and early 20th centuries. The seminal works of Faraday [21] and Mie [22] provided a scientific ground to the phenomenal attraction of metal nanoparticles and their unique optical properties. Noble metals - silver and gold in particular -, exhibit interesting optical properties at the nanoscale that differ markedly from those of the bulk material, and also from those of the isolated atomic constituents, due to the excitation of localized surface plasmon resonances (LSPRs) [23]. Following recent advances in the synthesis and surface modification of metallic nanocrystals [24], the ease of their integration into nanocomposites [25], and the control over their plasmonic properties [26], chiral metal nanostructures have emerged, and justify the present review. 
Whereas excellent reviews by Gautier and Bürgi [15] and Noguez and Garzón [27] have been specifically devoted to sub-nanometer metal clusters with inherent chirality and a strong associated optical activity, a report highlighting advances in the field of chiral plasmonic nanoparticles is still missing. This gap in the literature has occurred due to difficulties in the chemical synthesis of nanoparticles with intrinsic chiral morphology, or the reliable assembly of achiral particles into chiral superstructures [28]. Since the field of plasmonics is currently undergoing fast development, we intend to focus this review on the recent work with isotropic and anisotropic plasmonic nanoparticles, and only briefly mention chiral metal nanoclusters, placing each system within the context of the corresponding origin of the observed chirality. Future prospects in this field will also be discussed; we hope that through this perspective and the diversity of structures conveyed in this review article the reader will gain a greater appreciation of chirality in metal nanoparticles.

\section{Mechanisms for obtaining optical activity in metal nanoparticles}

As a general definition, any geometrical object is chiral or dissymmetric if its mirror image cannot be brought to coincide with itself [29]. Analogously, but expressed in terms of group theory, the criterion for an object or group of objects to be chiral is that it must not contain improper rotation axes, $S_{n}$, such as planes of reflection $\left(S_{1}=\sigma\right)$ and centers of inversion $\left(S_{2}=i\right)[30,31]$. These geometrical requirements make the measurement, and even the qualification, of chirality a non-trivial issue in many systems. It is rather intuitive to compare an object with its enantiomer to assess their degree of spatial overlap. In this way it seems easy to judge the chirality of macroscopic objects, like for instance one of our hands. However, defining an object as chiral or achiral is not straightforward when geometry changes occur with time, as in the case of organic ligands used to transfer chirality onto nanoparticles or dynamic self-assembled systems.

Going back to the uses of chirality at the nanoscale, many are related with the preparation of materials with optical activity. Circular dichroism (CD), defined as the difference in extinction of left and right circularly polarized light, is one of the most commonly used spectroscopic techniques when studying chirality. The optical activity of chiral systems is thus often measured through the anisotropy factor, also known as g-factor [32,33]:

$g=\frac{\Delta \varepsilon}{\varepsilon}$

where $\Delta \varepsilon$ and $\varepsilon$ are the molar circular dichroism and molar extinction, respectively. In this review, we will not only focus on a particular shape and/or spatial ordering of objects, but also on the efficiency of the sample to differentiate between left and right circularly polarized light, comparing the chirality of different example cases by means of their anisotropy factor.

A comparison of relevant chiroptical metal nanoparticles in solution (Fig. 2) shows some of the highest reported gfactors and their corresponding spectral ranges [34]. Within

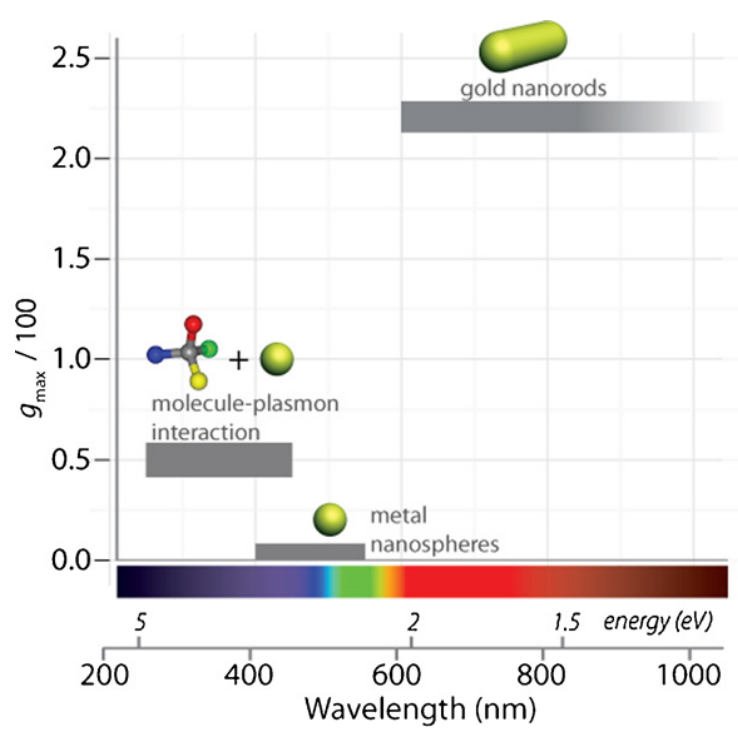

Figure 2 Representative values of chiroptical metal nanoparticles with strong anisotropy factors $\left(g_{\max }\right)$ and the corresponding typical spectral ranges of metal nanoparticles in solution. Reproduced with permission from Ref. [34]. Copyright (C2011 Wiley-VCH.

the UV region, the $g$-factor of organic molecules has been enhanced up to 0.005 by combination with sub-nanometer metal clusters [15]. Additionally, in the visible region metal nanospheres with surface-plasmon circular dichroism (hereafter termed SP-CD) at $\sim 400$ and $\sim 520 \mathrm{~nm}$ for silver and gold nanoparticles, respectively, have been shown to register $g$-values up to 0.001 [35-39]. Although several reports have dealt with optically active metal nanospheres in nonfluid systems, such as nanocomposite gels and films [40,41], there are no examples of high SP-CD of isotropic nanoparticles in fluid media. However, outstanding SP-CD values were recently reported for assemblies of anisotropic gold nanorods (NRs) with $\mathrm{g}$-factors as high as 0.02 covering a wide range of the vis/NIR spectrum [34]. This value is comparable with the highest $\mathrm{g}$-factor reported for organic molecules such as polyaromatic compounds (0.05) [42], alleno-acetylenic macrocycles and oligomers $(0.01)[43,44]$, and protein complexes $(0.06)$ [45].

Although in many examples the mechanisms behind optical activity in metal nanoparticles cannot be easily identified, their chiroptical activity may in principle be restricted to two distinct origins: (i) nanoparticles with individual chirality, and (ii) collective interactions between 3D ordered nanocrystals. Examples pertaining to these two mechanisms will be more deeply considered and analyzed in the following sections.

Within the generic class of individual chirality, three main types can be identified (Fig. 3) [15]: (a) the presence of chiral ligands can favor the growth of an intrinsically chiral core; (b) for achiral cores, optical activity can be induced by a chiral shell through vicinal effects or through a chiral electrostatic field; and (c) in an originally achiral core the relaxation of the surface atoms involved in the adsorption of the chiral ligand may create a chiral footprint. Most of the chiral nanoparticles reported to date with individual dissymmetry have been mainly sub-nanometer clusters (see next 

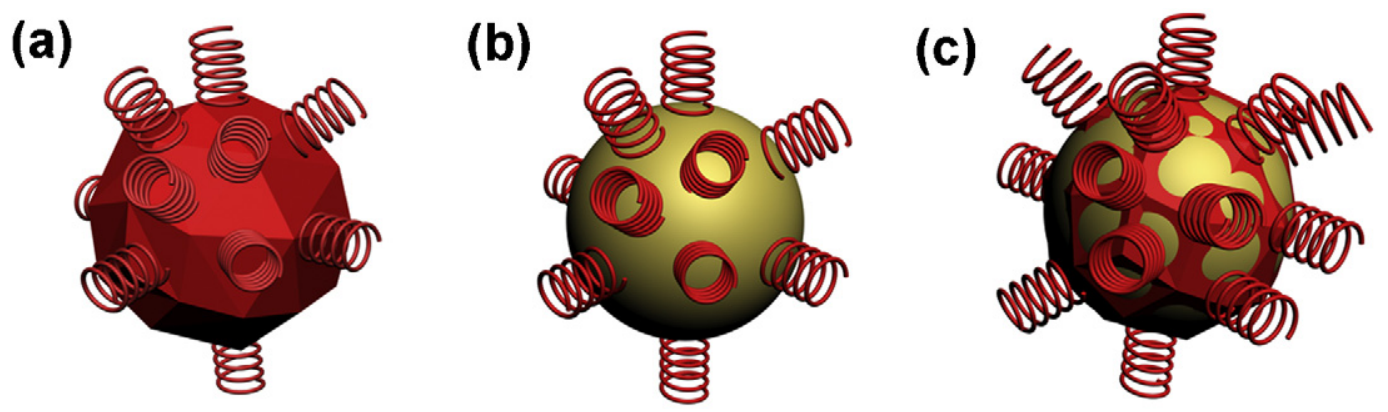

Figure 3 Schematic view of different origins of optical activity observed for metal particles with individual chirality. (a) Intrinsically chiral nanoparticle. (b) Chiral distribution of nanoparticle electron density induced by molecular chiral ligands. (c) Chiral footprint on the nanoparticle surface. Chiral capping ligands are represented by red helices. The red color at the nanoparticle surface indicates regions with intrinsic chirality.

Section Nanoparticles with individual dissymmetry); their chirality originating from combinations of (a), (b), and/or (c) mechanisms. The corresponding CD responses have been restricted to the UV region of the spectrum (outside the usual regime of LSPRs), with relatively low optical activity efficiencies (Fig. 2).

On the other hand, the origin of collective chirality is based on interactions between nanoparticles assembled with 3D chiral order. This mechanism has been recently proposed as the origin of chirality in plasmonic nanoparticles of different size and composition, providing already after only few examples record SP-CD responses in the vis-NIR region of the electromagnetic spectrum (Fig. 2). Although a deeper insight is needed to fully understand the collective mechanism in metal nanoparticles (see Section Plasmon hybridization and optical activity), the analogy with exciton-coupling theory can be useful in visualizing this phenomenon $[32,34,46]$. In this well-established theory used in molecular systems, the coupling between two identical chromophores is reflected in a split or broadened absorption band, centered at the characteristic absorption wavelength of the isolated chromophore. If the two chromophores are not coplanar a bisignated CD couplet is generated with two opposite Cotton effects (i.e. dichroic bands) (Fig. 4a-d) $[32,33,47]$. The intensity and, more importantly, the sign of the couplet, defined by the sign of the longer wavelength Cotton effect, are dictated by the relative orientation of the two chromophores. By analogy, metallic nanoparticles may be considered as interacting dipoles that are able to display optical activity when organized in 3D chiral superstructures. As a result, the characteristic bisignated signature of SP-CD in plasmonic metal nanoparticles is strongly reminiscent of exciton-coupling in molecules with coupling between two identical chromophores (Fig. 4e) [41]. However, as discussed below, the exact mechanism behind collective optical activity often remains unclear, and more than one mechanism may concurrently affect the response of a specific system.

\section{Nanoparticles with individual dissymmetry}

We consider in this section optically active small metal nanoparticles (quantum size nanoclusters) with no distinct LSPR. Metal nanoclusters constitute an important inter- mediate size regime between localized atomic states in molecules and delocalized band structures in plasmonic nanoparticles [48]. For diameters below $\sim 2 \mathrm{~nm}$ (several tens of atoms), particles sustain molecule-like optical transitions that depend on the compositional number of atoms and display so-called superatom properties [49]. As nanoclusters grow larger, the molecule-like properties develop into an optical absorption band stemming from the LSPR of their free conduction electrons [50].

Unlike in clusters, the generation of individual dissymmetry in plasmonic metal nanoparticles has proven so far an elusive pursuit, in which only a limited number of successful examples have been reported so far (see Section Collective chirality in plasmonic nanoparticles). The main difficulty for the preparation of such nanocrystals is directly related to their inherent reactivity [24]. Chiral imprinting of ligands during the growth of the nanocrystal cannot be controlled to obtain a pure enantiomeric colloidal dispersion, especially for particles larger than $10 \mathrm{~nm}$. Indeed, there are no convincing examples of mechanism (a) in the literature for this size range, while mechanisms (b) and (c) have been proposed as the main origin of chirality for such plasmonic systems (Fig. 3) [51,52]. In an attempt to circumvent these intrinsic difficulties, the search for approaches toward collective chirality has therefore represented the major driving force for research on chirality control with plasmonic nanoparticles.

Silver nanoclusters: Typically, single stabilized silver nanoclusters are prepared using in situ one pot methods that involve the reduction of silver salt precursors with mild reducing agents in organic solvents, in the presence of stabilizing ligands with thiol or amino anchoring groups [15]. In general, such clusters exhibit well-defined absorption peaks in their UV-vis spectra and characteristic CD bands in the visible range when the stabilizing ligands are chiral. Recently, Cathcart and co-workers prepared clusters (22-28 silver atoms) that display different CD features depending on the capping agent used, with opposite sign for captoril and glutathione ligands (Fig. 5a) [53]. Such differences have been attributed to the higher affinity of captoril ligands for the cluster surface, which are able to further imprint their chirality during cluster growth (mechanism (a)) [54]. Therefore, using a combination of both chiral ligands with essentially opposite CD signs enables tuning of chirality in silver clusters. The same origin has 

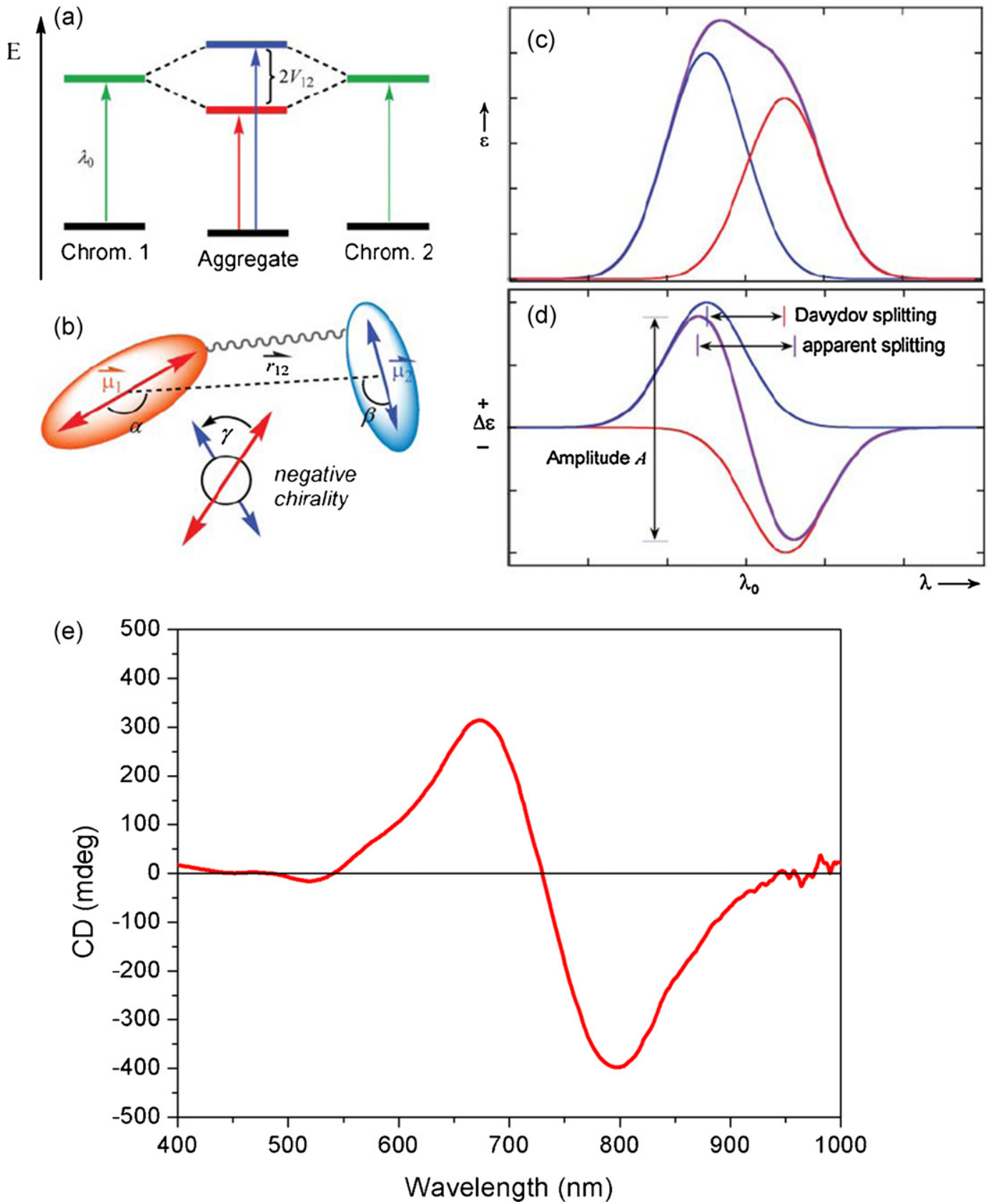

Figure 4 (a) Splitting of the excited states of two degenerate exciton-coupled chromophores linked by a chiral spacer. (b) Definition of geometrical parameters necessary for predicting CD sign and intensity [32]. Expected absorption (c) and CD spectra (d) in case of exciton-splitting as shown in (a): component spectra thin lines in blue/red, resulting spectra thick lines in purple. The magnitude between minimum and maximum for the split CD spectrum is called amplitude $A$. The two transitions are separated by an energy $2 V_{12}$, called Davydov splitting [32] - Reproduced with permission of The Royal Society of Chemistry. (e) Bisignated CD spectrum of gold nanorods ordered with collective chirality [34].

been reported to be the main factor behind the optical activity of L- or D-penicillamine-protected silver nanoclusters [55]. An interesting recent development based on a chiral induction strategy from achirally modified silver nanoclusters was reported by Kimura and co-workers [56]. They synthesized nanoclusters protected with achiral 3mercaptophenylboronic acid (mean core diameter $0.8 \mathrm{~nm}$ ) that showed $C D$ signals (at $\sim 350 \mathrm{~nm}$ ) in the presence of $L$ - or $D$-fructose, with a mirror-image relationship at the UV transitions (Fig. 5b). A comparison between the chiroptical properties of this system ( $g$-factor $3.2 \times 10^{-5}$ ) and penicillamine-protected silver nanoclusters ( $g$-factor $1.0 \times 10^{-3}$ ) points toward a dissymmetric field of the phenylboronic acid-fructose complex, as the mechanism behind the induced optical activity [57] (mechanism (b)).

Gold nanoclusters: These particles have attracted a large interest during the past couple of decades [58]. Since Schaaff and co-workers discovered intense Cotton effects 

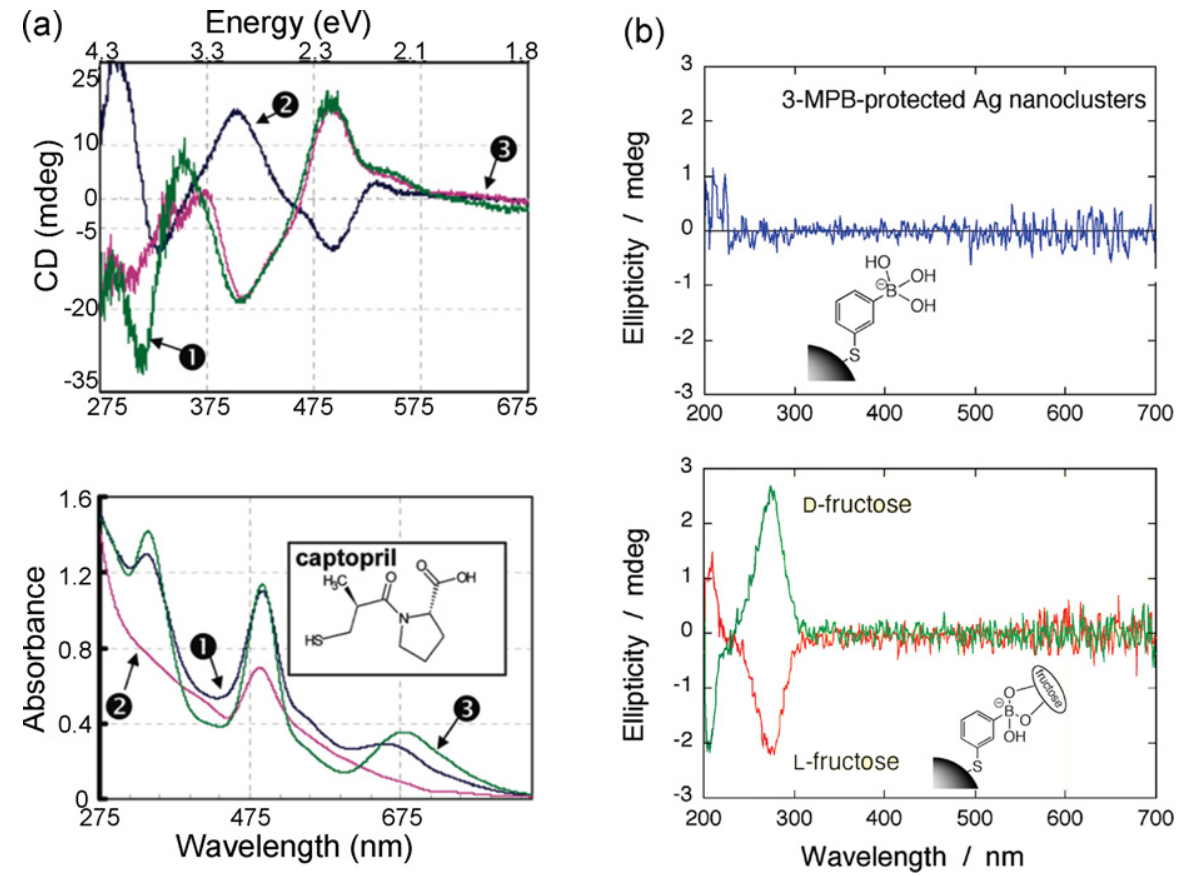

Figure 5 (a) Circular dichroism spectra (top) and UV-vis spectra (bottom) of representative silver nanoclusters stabilized using different ligands (1: captoril; 2: glutathione; 3: captoril and glutathione (molar ratio 62:38)). Reproduced with permission from Ref. [53]. Copyright (2010 American Chemical Society. (b) Circular dichroism spectra of silver clusters coated with 3-mercaptophenylboronic acid (top) and their complexes with L- or D-fructose (bottom). The insets show schemes of the cluster structures.

Adapted with permission from Ref. [56]. Copyright @2010 American Chemical Society.

in the metal-based electronic transitions of size-selected glutathione-passivated gold nanoparticles ( 25 gold atoms) $[59,60]$, their optical activity - in particular the origin of chiroptical effects - has been extensively investigated, both theoretically and experimentally. These works on $\mathrm{Au}_{28}(\mathrm{SG})_{16}$ (SG = glutathione) and $\mathrm{Au}_{14}(\mathrm{MTI})_{6}(\mathrm{MTI}=\mathrm{R}$-methylthiirane) systems, using a charge-perturbed particle-in-a-box model and time-dependent density functional theory (TDDFT) calculations, supported the hypothesis that a chiral field arising from the adsorbates can induce a chiroptical signature in achiral metal clusters (mechanism (b)). Indeed, theoretical $C D$ spectra of undecagold-bidentate phosphine complexes indicated that the optical activity of the metal core is very sensitive to the chiral arrangement of the surrounding ligands [61]. On the other hand, studies by Garzón and coworkers suggested that the lowest energy structures of some bare gold clusters are chiral [62,63]. However, they also found nanocluster structures with an approximate center of symmetry $\left(\mathrm{Au}_{25}(\mathrm{SR})_{18}, \mathrm{SR}=\mathrm{SCH}_{2} \mathrm{CH}_{2} \mathrm{Ph}\right)$ that are therefore achiral.

Regarding cluster preparation, various types of molecular gold clusters have been isolated, such as $\mathrm{Au}_{10}$, $\mathrm{Au}_{15}, \mathrm{Au}_{18}, \mathrm{Au}_{22}, \mathrm{Au}_{25}, \mathrm{Au}_{29}, \mathrm{Au}_{33}$, and $\mathrm{Au}_{39}$ [64]. Among them, $\mathrm{Au}_{25}$ has been found to be particularly stable. Thiolate ligands can be easily adsorbed on $\mathrm{Au}_{25}$ clusters generating a local chiral environment near the metal surface (mechanism (c)), but also transferring chirality onto the electronic structure of the achiral metal core (mechanism (b)). As expected, $\left[\mathrm{Au}_{25}\left(\mathrm{SCH}_{2} \mathrm{CH}_{2} \mathrm{Ph}\right)_{18}{ }^{-}\right]$ $\left[\mathrm{TOA}^{+}\right](\mathrm{TOA}=$ tetraoctylamonium) cluster is optically inac- tive. Upon exchange with BINAS (1, 1'-binaphthyl-2,2-dithiol) or NIC (N-isobutyryl-cysteine), intense bands were observed in the $C D$ spectra, with mirror-image for clusters covered by the corresponding enantiomeric ligands (Fig. 6) [65]. The registered spectra certainly underlined that the degree of optical activity is larger after exchange with BINAS, as compared to exchange with NIC. Indeed, the maximum anisotropy factor induced by BINAS was more than five-fold larger than that induced by NIC. This result demonstrated that optical activity in gold clusters may be ligand-dependent. Interestingly, for both cases discussed above, the anisotropy factor was reported to be larger (by a factor of 3) for particles directly prepared with the respective thiols, than for those obtained via the ligand exchange method. Thus, apparently only a few chiral ligands are needed to induce significant optical activity in the metalbased electronic transitions $[63,66]$. All these observations are consistent with a chiral footprint model (mechanism (c)). Moreover, Murray and co-workers have recently shown that the $\mathrm{Au}_{25}(\mathrm{GS})_{18}$ cluster adopts a two-shell structure that is an icosahedral $\mathrm{Au}_{13}$ kernel with the remaining 12 gold atoms forming an outer gold shell [67], with a net charge of -1 , as shown by means of NMR in combination with mass spectrometry [68]. The chiral optical signals in the visible region from the $\mathrm{Au}_{25}(\mathrm{SG})_{18}$ clusters seem to be affected by the chiral ligands but not from the chiral $\mathrm{Au}_{25}$ core (mechanism (b)), since the NMR spectra did not show any core-dependent peak splitting.

Interestingly, TDDFT calculations have predicted a nonzero $C D$ spectrum for single $\left[\mathrm{Au}_{25}(\mathrm{SR})_{18}{ }^{-}\right] \quad(\mathrm{R}=$ achiral 

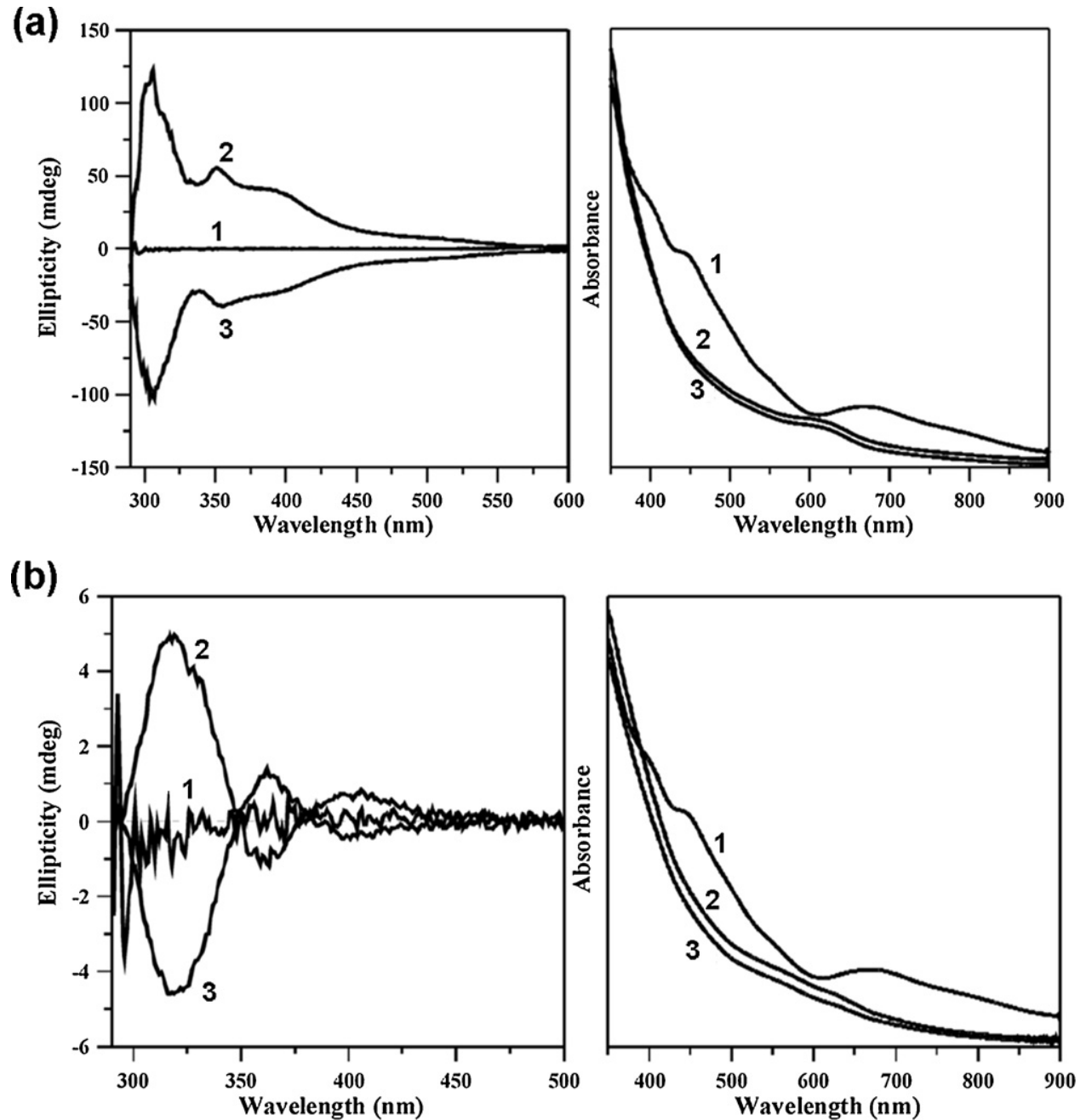

Figure 6 (a) Circular dichroism (left) and UV-vis (right) spectra of $\mathrm{Au}_{25}$ before (1), and after exchange with R-BINAS (2) and S-BINAS (3). (b) Circular dichroism (left) and UV-vis (right) spectra of $\mathrm{Au}_{25}$ before (1), and after exchange with L-NIC (2) and D-NIC (3).

Adapted with permission from Ref. [65]. Copyright @2010 American Chemical Society.

methylthiolate) nanoclusters. However, a zero CD spectrum was measured when achiral ligands were used. This finding was explained on the basis that only chiral ligands possess the enantioselectivity needed to break the symmetric environment in the racemic mixture of chiral ligand-protected metallic clusters. Thus, the induced dissymmetry was responsible for the observed optical activity [69].

Vibrational circular dichroism: Adsorption of molecules on nanocluster surfaces is of fundamental importance for many processes involving separation, bio-sensing, surface processing, lubrication, and heterogeneous catalysis [70]. Thus, the conformation of molecules at the surface may have a pronounced effect on the physicochemical properties of particles. However, obtaining information about the surface organization at the molecular level is often rather difficult, and the use of spectroscopic sensing probes, such as chromophores [71] or Raman active molecules [72], has resulted very useful. In this context, vibrational circular dichroism (VCD) - differential absorption of left- and right-circularly polarized light in the infrared region -, has emerged as a sensitive technique for the study of the conformational organization of chiral capping agents onto nanocluster surfaces [57].

Whereas the size of metal nanoclusters has a strong influence on the UV-vis and CD response, this parameter has less remarkable effects on IR and VCD spectra. Indeed, particles with different sizes display very similar VCD spectra. This shows that the vibrational behavior of the adsorbed molecules can be used to study the molecular conformation of ligands [57]. Models of bidentate ligands, such as BINAS or $\mathrm{NIC}$ adsorbed on $\mathrm{Au}_{10}$ or $\mathrm{Au}_{8}$, respectively, were used to unequivocally determine the conformation of ligands at the cluster surface (Fig. 7) [73]. Analysis of the lowest energy conformers showed that the ligands are adsorbed via two anchoring groups that are attached to two gold atoms in a bridge site, one of the two gold atoms being shared between both groups. Bürgi and co-workers used VCD combined with density functional theory (DFT) calculations to study the conformation of NIC adsorbed on $\mathrm{Au}_{8}$ clusters [74]. This study further demonstrated the potential of VCD spectroscopy for structure elucidation of chiral molecules adsorbed on metal particles. 

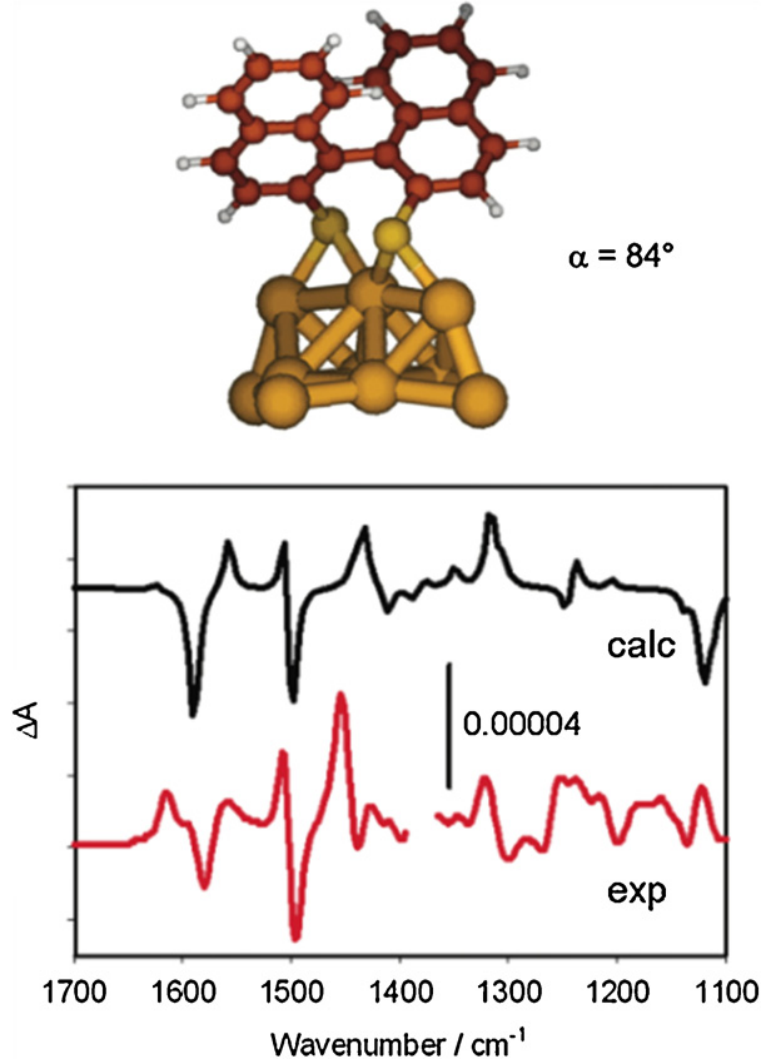

Figure 7 Calculated conformer of R-BINAS adsorbed on a $\mathrm{Au}_{10}$ cluster. Simulated (black) and measured (grey) spectra of RBINAS adsorbed on a nanocluster.

Reproduced with permission from Ref. [65]. Copyright (02010 American Chemical Society.

Individual chiral plasmonic nanoparticles: It has been observed that the $g$-factor of chiral nanoclusters increases with decreasing particle size [15]. For small clusters, most of the metal atoms are located at the surface, directly in contact with the chiral adsorbates, thus providing higher efficiencies of chiral induction. Therefore, the preparation of dissymmetric plasmonic nanoparticles has been limited to sizes close to the nanocluster limit $(5-10 \mathrm{~nm})$.

Govorov and co-workers recently reported an interesting study in which peptides with differing secondary structures (random coil and $\alpha$-helix) induce optical activity in $10 \mathrm{~nm}$ gold nanoparticles (Fig. 8a). The peptide-nanoparticle interactions produce an SP-CD signal at the LSPR wavelength $(\sim 520 \mathrm{~nm})$ that was attributed to strong dipolar interactions between both components (mechanism (b)) [75]. This novel result concerning plasmonic nanoparticles had been theoretically predicted by the same group [52], suggesting that chiral electromagnetic currents were generated inside the metal nanocrystals due to the perturbing presence of chiral chromophores (Fig. $8 \mathrm{~b}-\mathrm{c}$ ). This origin was proposed as the main contribution to the SP-CD signal when the absorption band of chromophores is well separated from the LSPR. Additionally, they showed that achiral plasmonic nanocrystals could enhance the $C D$ of chiral molecules coupled via dipole and multipole Coulomb interactions. As an example, proteins coating gold nanoparticles have been shown to present significant CD enhancements [76]. In a similar study, Katz and co-workers reported that gold nanoparticles $(\sim 5 \mathrm{~nm})$ modified with chiral 1,3-disubstituted diamino-calix [4]arene ligands show SP-CD bands $(\sim 520 \mathrm{~nm})$ and CD enhancement ( $\sim 10$-fold) of the macrocyclic ligands [51].

\section{Collective chirality in plasmonic nanoparticles}

Solutions of metal nanoparticles obtained by colloidal synthesis are optically inactive - either the particles do not display intrinsic chirality, or they form a racemic mixture. The characteristic signature of LSPR(s) can be readily observed by UV-vis spectroscopy as strong extinction peak(s) [77]; however, a measurement of circular dichroism or optical rotation would reveal no trace of optical activity. This is because the large number of particles interacting with the light beam averages out any local signature of individual chiral particles, or because of the short-lived formation of chiral aggregates [78]. Up to now, due to synthetic limitations, no examples have been reported of colloidal metal nanoparticles in the plasmonic size regime, from few to hundreds of nanometers, displaying an intrinsic chiral structure. Chirality on such systems has mainly originated from dipolar interactions with active capping agents [75] or observed in organized assemblies of particles [28]. The preparation of a colloidal dispersion of such imaginative dissymmetric nanocrystals (Fig. 9a) will certainly demand a high degree of control on synthetic chemistry that is closer to that achieved in chiral organic reactions [79] than what is currently available in colloid-chemical synthesis [24]. In fact, state-of-art methods used to produce single particle chirality at the nano- and micron-scale on substrates are nanoimprint lithography [80], and electrochemical deposition of metals on templates [81]. Although a wide variety of wet chemistry-based synthetic methods are currently available for the preparation of metal nanoparticles within narrow size and shape distributions [82], the controlled incorporation of chirality during nucleation and growth of nanocrystals has remained impractical and inaccessible to the current state of colloid chemistry. These approaches would exceed the shape-controlled synthesis of anisotropic particles, such as nanorods [83], nanowires [84] or nanostars [85], meaning the precise access to specific reactive sites in the crystallographic structure, with remarkable differences in energy [86] that induce an anisotropic growth of branches, nanoparticle junctions, and/or twists in controlled directions (Fig. 9a) [87].

Meanwhile, the need to circumvent the synthetic limitations with plasmonic nanoparticles has been a driving force at the forefront of chirality and nanotechnology, and has prompted the use of directed-assembly strategies [88] to generate stable chiral 3D assemblies (Fig. 9b). These organizations of particles resemble the geometry of: (i) chiral molecules, where nanoparticles with different composition, size or shape can be linked through covalent bonding [89]; and (ii) chiral polymers, wherein metal nanoparticles are linked chemically or physically to chiral templates [34]. The collective chirality of such organized nanostructures is directly associated with their SP-CD responses at the LSPR of the metal due to interparticle plasmonic dipole-dipole interactions [90]. The choice of material is therefore important, and silver has been used more often than gold due 
(a)
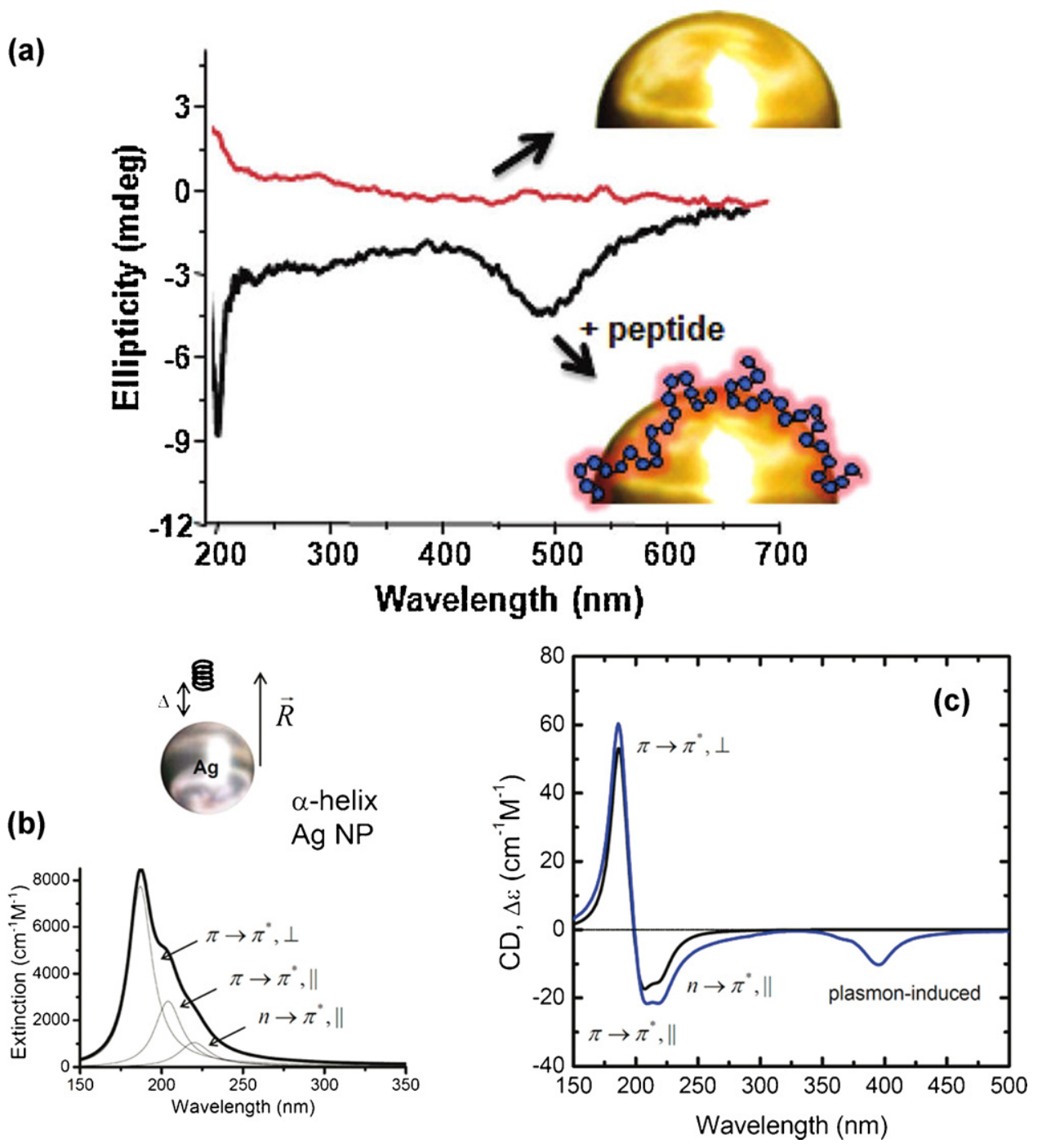

Figure 8 (a) CD spectra of gold nanoparticles in the presence (black) and absence (red) of chiral peptides. Reproduced with permission from Ref. [75]. Copyright @2011 American Chemical Society. (b) A model of interacting Ag NP and helix. Theoretical fit to the extinction of the ligand. The molar extinction is given for one chromophore. (c) Calculated CD signals for the ligand (black line) and for the nanoparticle-molecule complex (blue line) shown in (b); nanoparticle radius $=10 \mathrm{~nm}, \Delta=7 \mathrm{~nm}$, and $R=17 \mathrm{~nm}$. Reproduced with permission from Ref. [52]. Copyright @2010 American Chemical Society.

to its higher plasmonic efficiency [91], derived from a good separation between intrinsic interband transitions and LSPR modes, which results in narrower and more intense plasmon resonances.

Pseudo-random aggregation: Arguably the first reported examples of metal nanoparticles with optical activity dealt with the preparation of fractal aggregates of silver colloids [92]. The observed optical response was associated with the handedness of resonant plasmon modes registered by aggregates of interacting particles when their sizes are comparable to or larger than the wavelength of visible light [78]. However, this optical observation has been limited to micron-sized fractal objects, but was not detected in macroscopic random media containing nanoparticle aggregates, which are characterized by uncontrolled aggregation involving non-desired local fluctuations in the optical response of the material. This limitation demanded an improvement in the chiral organization of metal nanoparticles using directed assembly to obtain macroscopic systems with amplified optical activity, which has progressed significantly through the synthesis of metal nanocrystals with specific surface functionalities [70].

Nanoparticle linkage: DNA functionalization has been a popular choice for the stabilization of metal nanoparticles in bioimaging and biosensing applications during the last decade $[93,94]$. The interest stems from the high control over the design and synthesis of DNA sequences on the surface of nanoparticles [95], which resembles stereochemical control in organic chemistry [79]. Thus, DNA has been employed for building metal nanocrystal assemblies with different geometries, such as linear [96], triangular [97], pyramidal [98] and octahedral [99] configurations. Such engineered nanostructures have constituted the basis behind the preparation of metal nanoparticle chiral 
(a)

(b)
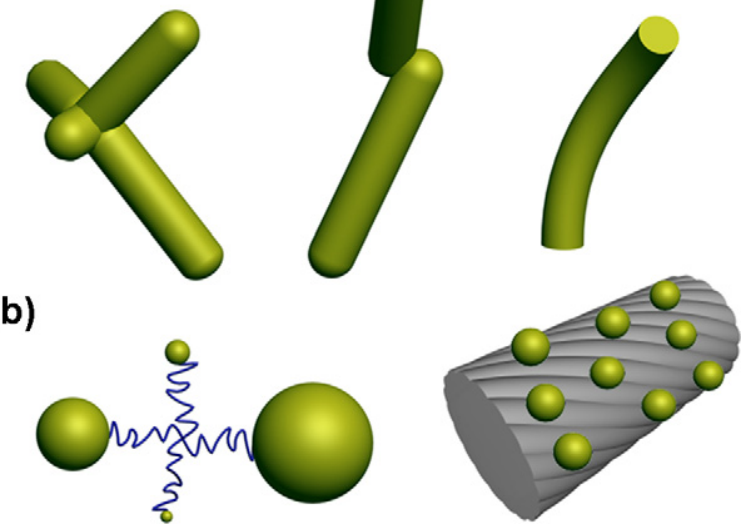

Figure 9 (a) Proposed structures of metal nanoparticles with individual chirality (from left to right, dissymmetric tetrapod, junction of rods, and pseudo-helix nanostructures). (b) Schematic organization of metal nanoparticles with collective dissymmetry resembling the chirality of molecules (left) and chiral polymers (right).

assemblies. Alivisatos and co-workers recently created discrete pyramids of double-stranded DNA (dsDNA) with gold nanospheres at the pyramid tips in solution [89], which have higher rigidity than conventional linear DNA [100]. In the pyramid design, each strand has a uniquely designed sequence that allows positioning of specific nanocrystals at each tip (Fig. 10a), using gold colloids of different sizes (5, 10,15 and $20 \mathrm{~nm}$ ) on different strands. The enantiomeric nanostructure was obtained by switching the position of two of these particles through conjugation with the opposite strand. An analogous method was employed by Kotov and co-workers, in which polymerase chain reaction was realized on the surface of plasmonic gold nanospheres as a tool for chiral self-organization with single-stranded DNA (ssDNA) [37]. The geometry of the products (ranging from dimers, trimers and tetramers to very complex agglomerates) was controlled through the density of DNA primer on the nanoparticles surface and the number of reaction cycles (Fig. 10b). Purification by gel electrophoresis was required to achieve optically active chiral assemblies, albeit with weak $C D$ signals at $650 \mathrm{~nm}$, therefore showing only the formation of a particular enantiomer that seems to arise from the specific chirality of DNA.

Chiral templates: In a different approach based on the use of biomacromolecules as templates with chirality, dsDNA was used to direct the formation of nanowires from gold nanospheres $(\sim 5 \mathrm{~nm})$ capped with poly-L-lysine [101]. The nanostructure resulted from attractive electrostatic interactions between positively charged nanocrystals aligned along both negatively charged strands at 1:1 DNA:nanoparticle mass ratio. At mass ratios larger than 1:5, nanoparticles cause strand separation, and subsequently DNA denaturation, which was confirmed by analysis of the CD signal from DNA. A variation of this method, where silver nanospheres were grown on covalently closed supercoiled dsDNA [102], produced important alterations in the plasmid conformation. The existence of weak SP-CD sig- nals in the wavelength region of plasmon resonances of silver spheres $(350-550 \mathrm{~nm})$ suggests formation of chiral organization of nanoparticles. Markovich, and co-workers have reported larger bisignated Cotton effects at the LSPR frequency ( $g$-factor below 0.001$)$ from silver nanoparticles grown on chiral dsDNA $[35,39]$, where silver nanospheres were produced by in situ reduction of $\mathrm{Ag}^{+}$bound to dsDNA. Although the proposed mechanism behind $C D$ induction was based on the assumption of the formation of a chiral metal core on the nanocrystal surface due to the influence of DNA, the use of a 3D chiral template and the bisignated feature of the $C D$ spectrum indicates that surface plasmon coupled CD between nanoparticles may also play a role. In fact, George and Thomas have shown that gold nanosphere aggregates grown onto the surface of $\mathrm{D}$ - and L-isomers of diphenylalanine nanotubes register moderate SP-CD ( $g$ factor below 0.001) (Fig. 11a) [36]. The resulting bisignated $C D$ signals at the LSPR frequency of gold nanoparticles were symmetrical mirror images for each enantiomeric template due to the dissymmetry transferred at the molecular level from the nanotubes onto the nanocrystals. In another example of chiral molecular transfer, silver nanoparticles were synthesized using thiol containing biomolecules (e.g., cysteine) [103], and associated through hydrogen bonding between biomolecules, resulting in generation of weak CD signals.

As DNA and molecular templates present chiral dimensions in the nanometer range, they necessarily limit the assembly to small metal nanoparticles (few nanometers). This usually leads to systems with low/moderate $g$-factors at the LSPR wavelength, thus limiting their uses and applications in plasmonics. As alternative templates, helical nanofibers have emerged as promising chiral platforms in which parameters such as diameter, length, and pitch can be precisely controlled from several nanometers up to microns. Recently, Leroux and co-workers have prepared insulin fibrils with diameters of $4-6 \mathrm{~nm}$, lengths of several microns, and helix pitches of $\sim 65 \mathrm{~nm}$ (Fig. 11b) [104]. These protein templates were incubated with silver ions in solution and then reduced to obtain nanosphere chains, which were structurally investigated by high-resolution atomic force microscopy and electron tomography. A 3D description of these fibrils with excellent contrast from the biomolecular and inorganic material was achieved using high-angle annular dark-field scanning transmission electron microscopy. The nanocrystals organized following the helical structure of the protein, and the nanocomposite showed a weak SP-CD band centered at the characteristic LSPR of silver nanoparticles. Notable improvements were obtained by $\mathrm{Li}$ and Liu [40] through in situ reduction of an organogel formed by an enantiomeric silver-coordinated compound, where chiral fibers of about $50-60 \mathrm{~nm}$ diameter and lengths of several micrometers were obtained. The size of the nanospheres on the organogel varied from 10 to $20 \mathrm{~nm}$, and showed a strong bisignated CD signal ( $g$-factor $\sim 0.02$ ) [105]. These authors also investigated the formation of chiral silver nanoparticles in ultrathin films of poly(methyl methacrylate), demonstrating that the construction of processable nanomaterials with chiroptical properties is possible.

Regarding the use of nanoparticles with different shapes, several recent examples deal with the preparation of 
(a)

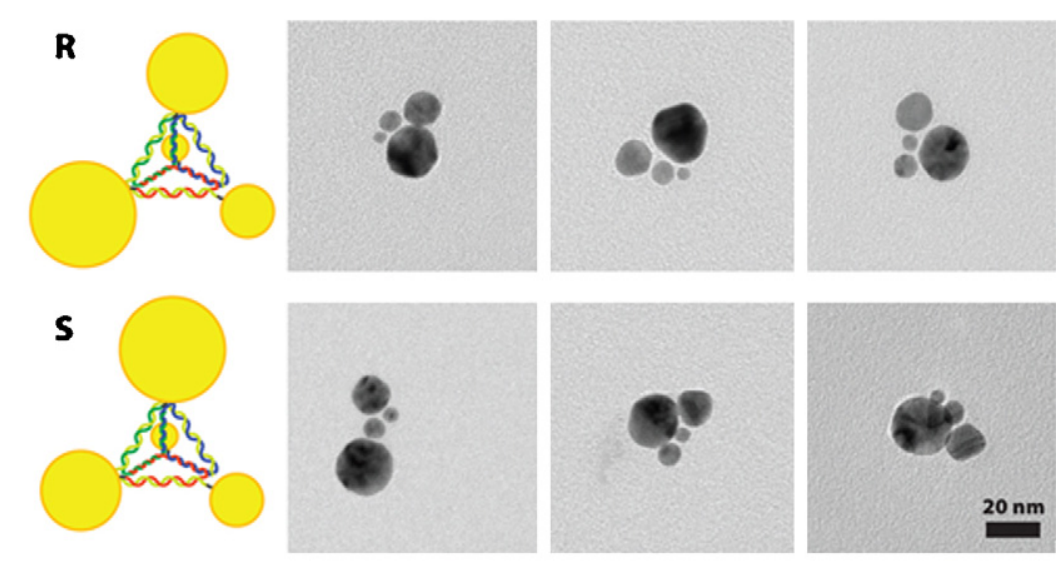

(b)

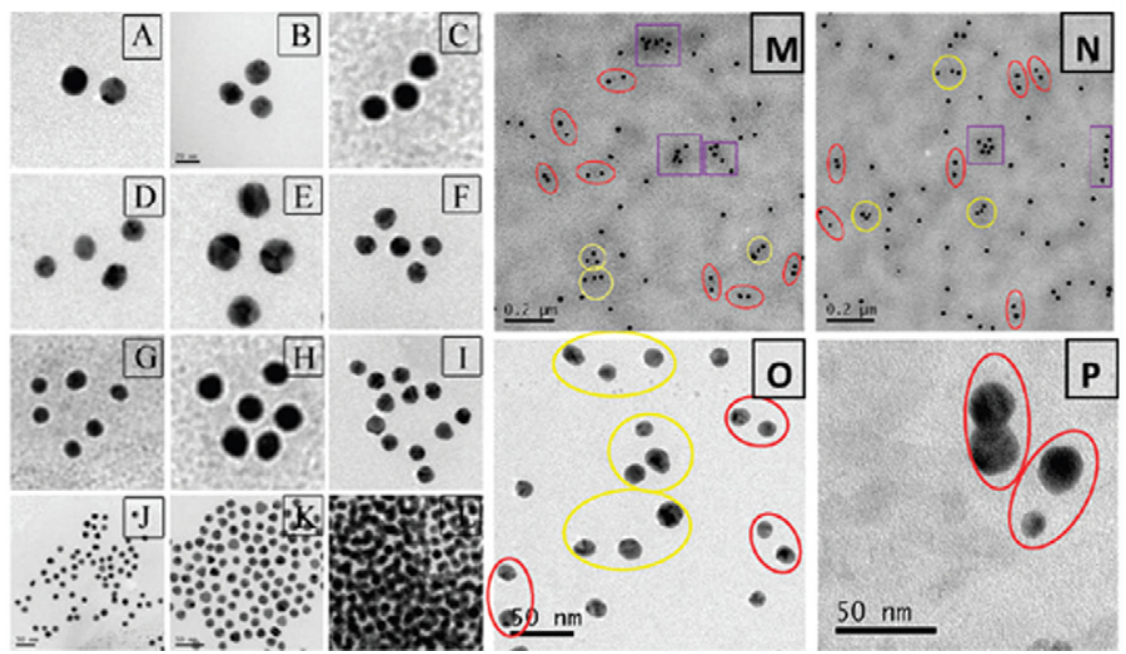

Figure 10 (a) Schematic representation and TEM images of chiral (R)- and (S)-pyramids made of gold nanospheres, organized by double-stranded DNA. Reproduced with permission from Ref. [89]. Copyright (02009 American Chemical Society. (b) TEM images of polymerase chain reaction products. $(A-L)$ Some characteristic self-assembled gold nanoparticles observed after an increasing number of reaction cycles. Representative TEM images describing the variety of self-assembled products after five reaction cycles with high $(M, N)$ and low $(\mathrm{O}, \mathrm{P})$ concentration of DNA primer on gold nanoparticles. Dimers, trimers, and multimers are marked by red, yellow, and purple lines, respectively. Reproduced with permission from Ref. [37].

Copyright $@ 2009$ American Chemical Society.

anisotropic gold nanoparticles using nanofibers as dissymmetric templates. Although silver displays better optical properties than gold [91], the higher chemical stability of gold nanostructures under colloidal synthesis conditions has favored their preferential application. Prasad and co-workers have reported the preparation of chiral nanocomposites with isotropic and anisotropic nanocrystals [41]. To produce this material, pre-made gold nanospheres ( $\sim 4$ and $\sim 10 \mathrm{~nm}$ ) were mixed with a polyfluorene and then annealed at $100-175^{\circ} \mathrm{C}$. Although the control of size and shape of the anisotropic nanoparticles generated upon annealing was still limited (non-spherical nanocrystals with sizes between 20 and $40 \mathrm{~nm}$ ), the supramolecular helical organization of the polymeric chains seemed to induce strong dipole-dipole interactions of the helically ordered nanoparticles, resulting in intense $g$-factor values $(\sim 0.02)$ in thin films [105]. Concurrently, our group recently pre- pared nanocomposites through the self-assembly of gold nanorods onto a scaffold made of supramolecular fibers with chiral morphology (Fig. 12a-d) [34]. The nanocomposites were obtained by mixing a fluid dispersion of fibers (with either right or left handedness) with gold nanorods coated with poly(vinylpyrrolidone). Both enantiomeric nanocomposites showed intense mirror-image SP-CD responses at the longitudinal LSPR wavelength ( $g$-factor higher than 0.02) (Fig. $12 \mathrm{e}-\mathrm{g}$ ). To understand the effect of the nanoparticle anisotropy, similar nanocomposites were prepared by assembly of gold nanospheres $(\sim 15 \mathrm{~nm})$. In this experiment no $C D$ signal could be recorded at the LSPR region. These results suggest that gold nanorods with $3 \mathrm{D}$ chiral ordering can be more efficient plasmonic nanoantennas than spheres for achieving intense and tunable SP-CDs, a finding that has been further modeled and analyzed $[34,46]$, as described in the following section. 
(a)
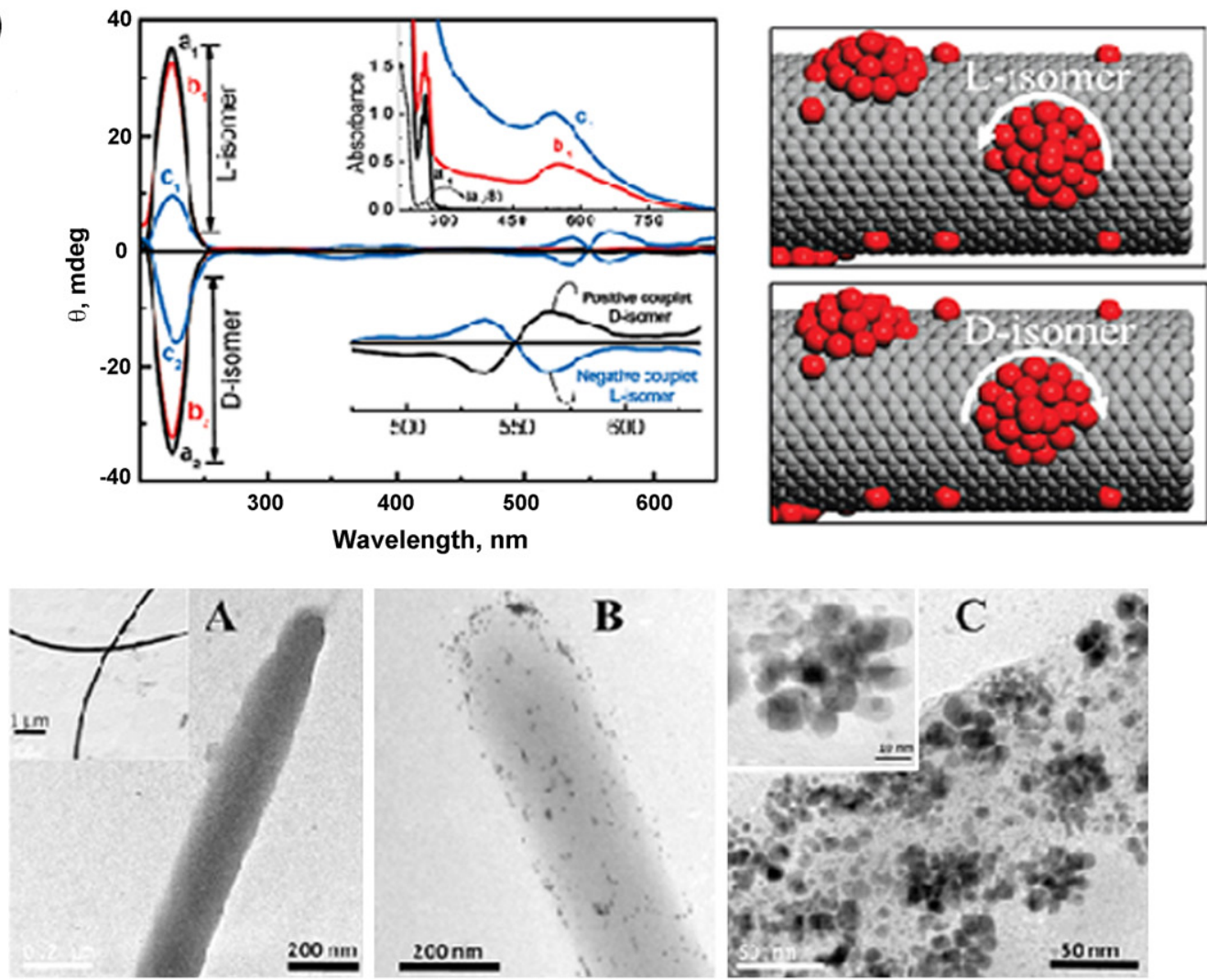

(b)
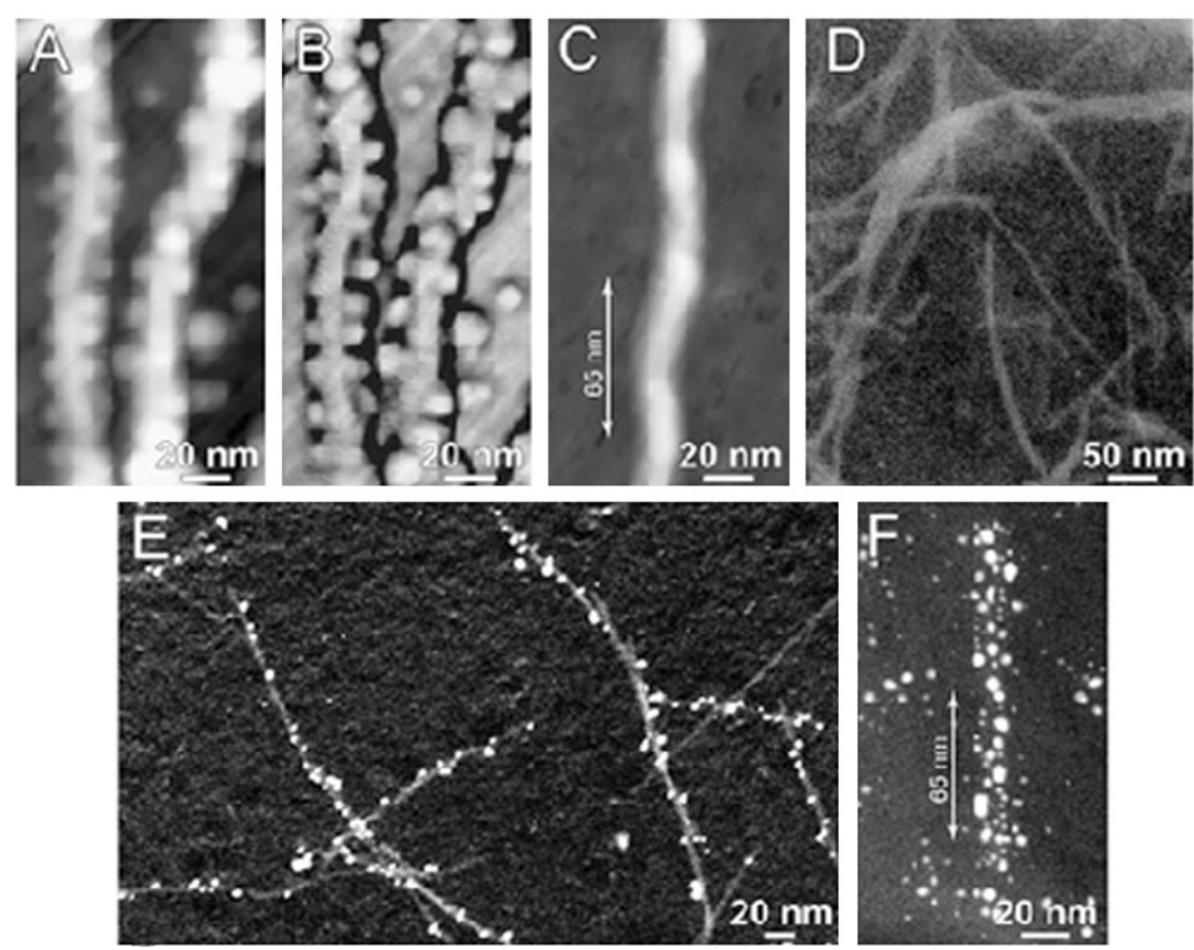

Figure 11 (a)Top: spectroscopic features in aqueous solution (CD spectra and absorption, inset) of chiral diphenylalanine peptide nanotubes, $a_{i}$; gold nanoparticle coated derivatives before $\left(b_{i}\right)$ and after $\left(c_{i}\right)$ photochemical irradiation of $a_{i}$ in the presence of gold salt precursor, $c_{i}$; ( 1 and 2 are the L- and D-isomers, respectively). Bottom: TEM images of peptide nanotubes in the absence (A) and presence (B) of nanoparticles, and after photochemical irradiation. Adapted with permission from Ref. [36]. Copyright $\bigcirc 2010$ American Chemical Society. (b) Topography (A) and phase (B) AFM images of fibrils coated with silver nanoparticles. AFM (C) and HAADFSTEM (D) images of uncoated fibrils, and STEM image of a silver-coated fibril (E). The helix pitch was determined to be $\sim 65 \mathrm{~nm}(\mathrm{~F})$. Adapted with permission from Ref. [104]. Copyright @2010 Wiley-VCH. 

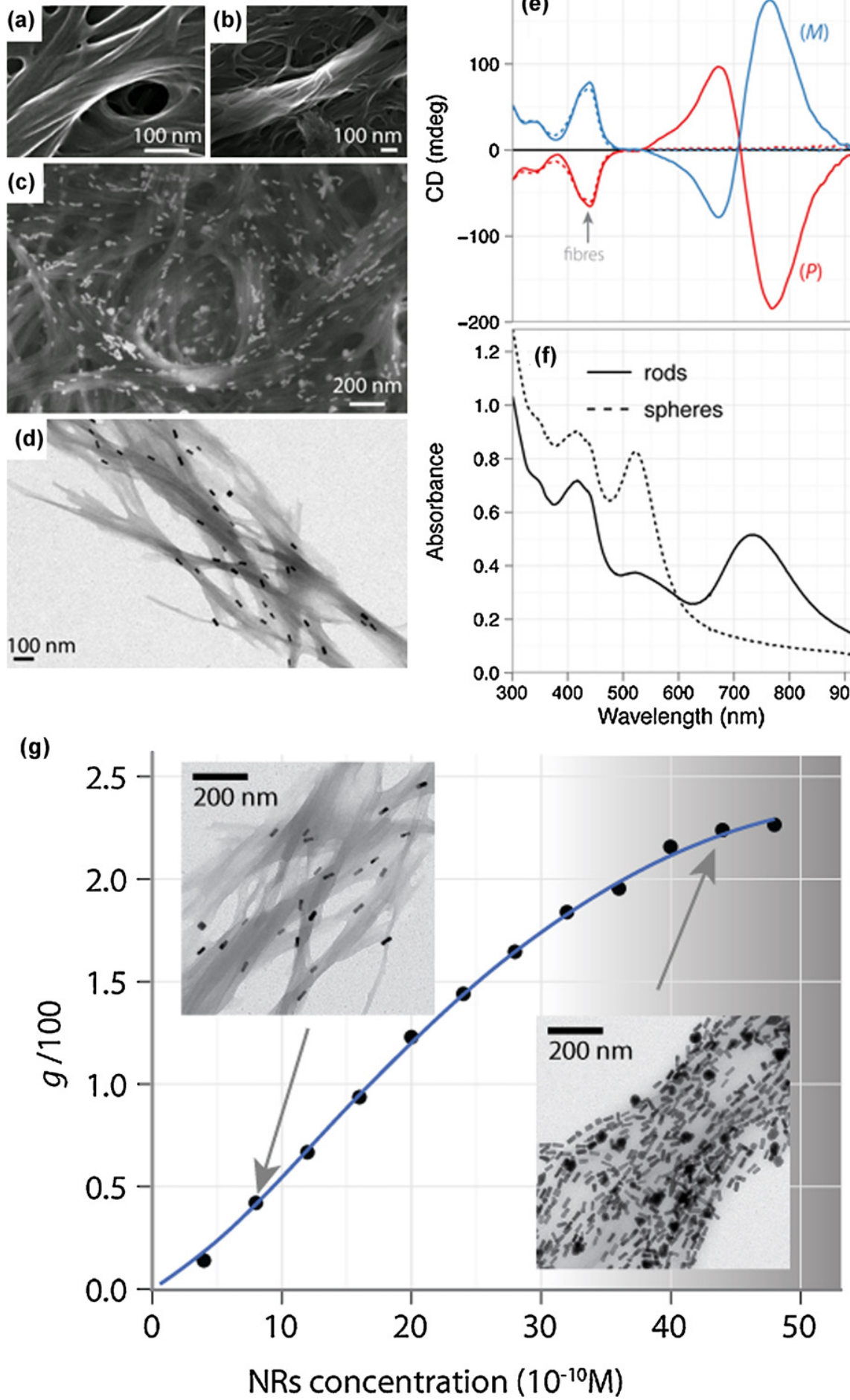

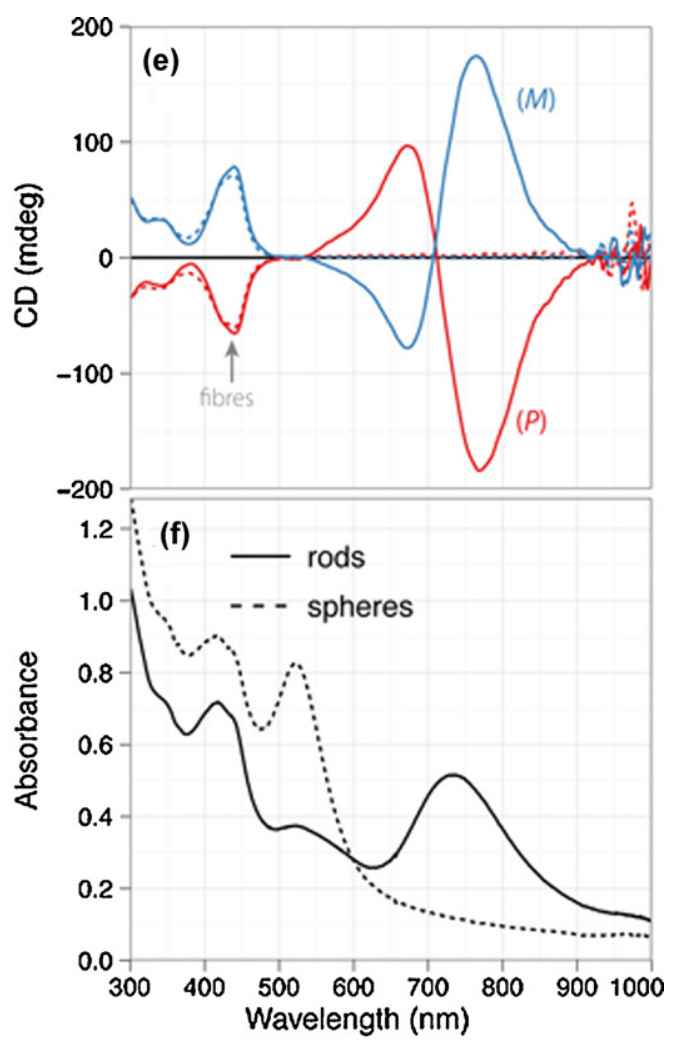


(a)

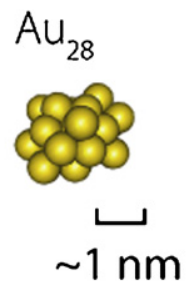

\section{Atomic cluster Absorption bands}

(b)

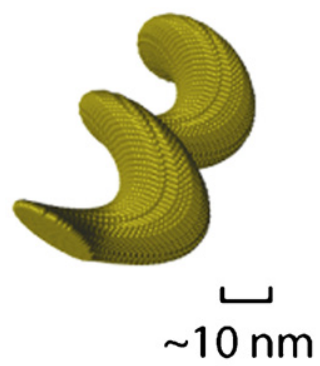

\section{Nanoparticle \\ Plasmonic response}

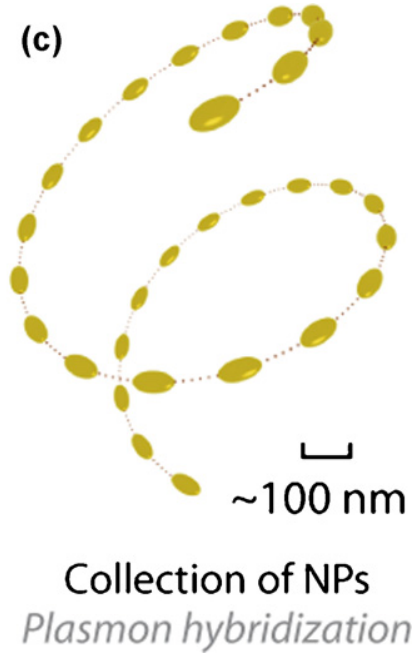

Figure 13 Pictorial representation of the structures where the coupled-dipole approximation was used in CD calculations: (a) an atomic cluster $\left(\mathrm{Au}_{28}\right.$, adapted from Ref. [111]); (b) a nanoparticle (helix inspired from Ref. [113]); (c) a collection of nanoparticles (helix inspired from Ref. [34]).

\section{Plasmon hybridization and optical activity - a coupled-dipole approximation}

It is only recently that a geometrical route to plasmonic circular dichroism has been envisaged. These recent experimental results hold great promise for intense and tunable SP-CD, further supported by recent theoretical investigations based on a coupled-dipole approximation.

Coupled-dipole model: Modeling circular dichroism with plasmonic nanoparticles falls short of an obvious choice of a theoretical framework; instead, a variety of numerical methods are routinely used by different research groups, each with its own strengths and weaknesses. A wide range of theoretical tools has been developed to elucidate the optical activity of chemical compounds, often based on quantum chemistry methods [106]. However, plasmonic nanostructures highly depart from the usual characteristics and assumptions required for such calculations. The number of atoms in a typical colloidal particle is far too large to be treated directly by first principle calculations, thus a classical electrodynamics approach is often adopted in the plasmonics literature [107], solving the Maxwell equations for continuous media with appropriate boundary conditions. Of particular value for its physical appeal and apt to treat a variety of systems is the coupled-dipole approximation [108-110], which offers a powerful and intuitive framework to treat classically the optical properties of metal clusters across a wide range of scales and arbitrary geometries. Fig. 13a illustrates the extent of the applicability of the coupled-dipole approximation in the theoretical

\section{(a) Chiral dimer of nanorods}

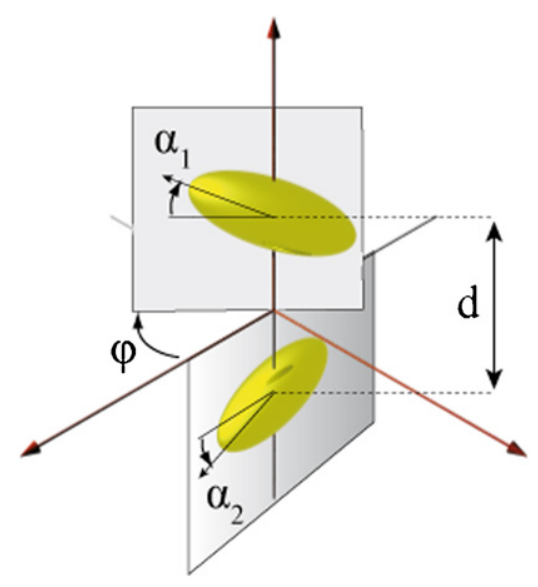

\section{(b) Exciton-coupling}

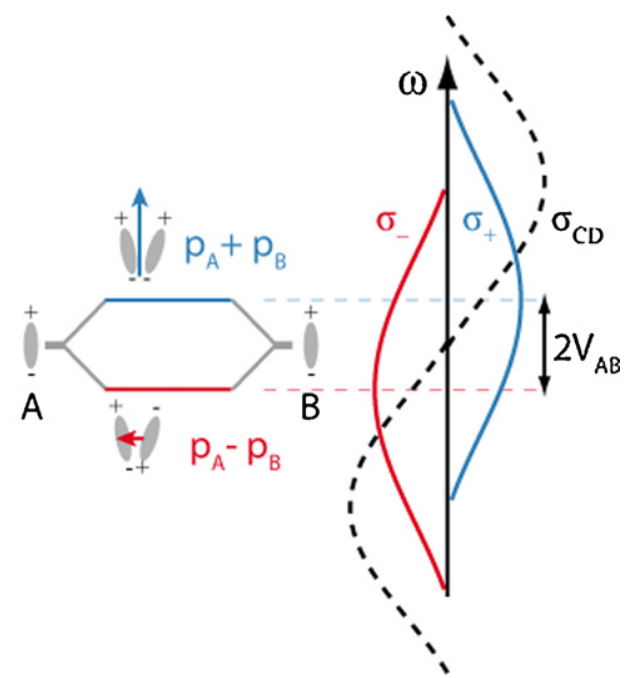

Figure 14 (a) Schematic representation of a chiral dimer of gold nanorods. (b) Illustration of the hybridization of LSPRs of two identical dipoles $p_{\mathrm{A}}$ and $p_{\mathrm{B}}$, and construction of the corresponding circular dichroism spectrum according to exciton-coupling theory. Adapted from Ref. [46]. Copyright @2011 American Chemical Society. 
treatment of circular dichroism in metal nanoparticles. Roman-Velazquez et al. [111] used the coupled-dipole approximation to investigate the CD of chiral clusters. In this form of the coupled-dipole approximation, each atom of the cluster is modeled as an electric dipole with an isotropic polarizability given by the Clausius-Mossotti formula:

$\alpha=\frac{a^{3}}{3} \frac{\varepsilon+1}{\varepsilon-2}$

where $a$ is the radius of a gold atom, and $\varepsilon$ the dielectric function of bulk gold. Optical activity arises from the intrinsic chirality of some clusters $[63,112]$. In Fig. 13b, we exemplify the discretization of a single nanoparticle into a large number of sub-units, where the optical response of the particle can be obtained by considering the self-consistent electromagnetic interaction between these meta-atoms. Such a calculation for optical activity was performed by Zhang and Zhao [113] for a silver helix. Finally, in Fig. 13c, the optical activity of a chiral assembly of plasmonic particles (here a helix) can be determined by approximating each particle as a triplet of electric dipoles [28,34]. This is the configuration we discuss in the remaining of this section.

The optical properties of a collection of interacting nanoparticles can be well described by a coupled-dipole model [114], provided that: (i) the particles have a size much smaller than the wavelength of light (typically $<100 \mathrm{~nm}$ ); and (ii) the interparticle separation is large compared to the particle size. The coupled-dipole approximation assumes a set of dipoles arranged at specific locations in space. Each nanoparticle is described by a $3 \times 3$ polarizability tensor, which can be rotated in a specific orientation [34]. In addition to the response of each individual dipole to the incident light, all dipoles are electromagnetically coupled via their scattered field. The self-consistent response of a group of dipoles to light is obtained by solving a linear system of coupled-dipole equations, with the incident electric field as a source term. From this formal solution of the scattering problem one can calculate the extinction cross-section $\sigma_{\text {ext }}(\omega)$ of the nanostructure in response to both left-handed and right-handed circularly polarized light. The difference between the two is defined as the circular dichroism crosssection $\sigma_{C D}=\sigma_{L}-\sigma_{R}[46]$.

$C D$ with a chiral dimer of gold nanorods: Arguably the simplest chiral system of plasmonic particles is formed by a dimer of nanorods separated by a certain distance, $d$ (Fig. 14a). The absence of a symmetry plane is ensured by a dihedral angle $\varphi$ different from 0 and 90 degrees. A chiral structure with spheres requires at least four equivalent spheres [28], and thus complicates the analysis. This archetypical system was in fact used by Kuhn [115] and Born [116] at the early stages of the molecular interpretation of the phenomenon of optical activity. Interestingly, such a dimer of plasmonic nanoantennas mimics the geometry of a molecule with two isolated chromophores [117] - a situation commonly described as exciton-coupling in organic chemistry (see previous Section Mechanisms for obtaining optical activity in metal nanoparticles) [118,119]. Using the more recent terminology of plasmonics and plasmon hybridization [117,120-122], we present in Fig. 14b a qualitative explanation of the origin of the circular dichroism in this structure [119].
Hybridization of LSPRs: Two identical gold nanoparticles widely separated (relative to their size and to the wavelength of light) support unperturbed eigenmodes of the electromagnetic field that describe the interaction of light with the scatterers in isolation. When the separation is reduced, the field that is scattered by one particle can excite the other particle and vice versa. This dynamical system describes a multiple scattering process, the result of which may be described by hybrid modes [123-127]. The optical properties of the ensemble are described by a linear combination of the two scatterers with a perturbation term that accounts for the relative interaction between the particles via their scattered field. The strength of the coupling between the two oscillators dictates the splitting of frequencies between the isolated mode and the new hybrid modes. Gold NRs present a very strong scattering response in the visible, strong particle-particle interaction can therefore be expected at short distances, a basic requirement for intense SP-CD. Due to the particular symmetry of the dipolar field, the coupling between two dipoles depends crucially on their relative position and orientation [128-130].

In the pictorial representation of Fig. 14b, the LSPR modes associated with two nanoparticles split into two hybrid modes with different energy levels (one blue-shifted, the other symmetrically red-shifted) when the dipoles are brought in proximity. The symmetric configuration yields a blue-shift of the resonance because the charges of the two particles acting in concert result in a stronger restoring force for the surface charge density associated with the LSPR. The antisymmetric configuration, however, leads to a red-shift of the LSPR. For exactly parallel dipoles with short separation, the antisymmetric mode will not couple to incident light (dark mode); however, this is not the case for chiral dimers which always have a non-zero net dipole moment.

Mimicking exciton-coupling: Focusing our attention on the contribution of the longitudinal LSPR mode, the system can be idealized as a dimer of two dipoles oriented along the long-axis of each particle. This is exactly the situation considered in exciton-coupling theory, in which chromophores (size $\sim 1 \mathrm{~nm}$ ) are replaced by nanoparticles. Light incident on the dimer causes each dipole to radiate an electromagnetic field, which in turn affects its neighbor. This dipole-dipole interaction is associated with an energy $V_{A B}$ separating the two energy levels corresponding to the symmetric $\omega_{+}$and antisymmetric $\omega_{-}$hybrid modes (Fig. 14b). The resulting CD spectrum can be obtained by summing the extinction band associated with each hybrid mode, with a strength and sign described by a rotational strength:

$R^{ \pm}= \pm \frac{\pi}{2 \lambda_{0}} R_{i j} \cdot\left(p_{i} \times p_{j}\right)$

which changes sign between the symmetric and antisymmetric hybrid modes ( $\lambda_{0}$ being the wavelength of the uncoupled LSPR, and $R_{i j}$ the vector joining the two dipoles $p_{i}$ and $\left.p_{j}\right)$. The construction depicted in Fig. 14b predicts a bisignated $C D$ spectrum, resulting from the difference between the extinction of each of the two eigenmodes.

This coupled-dipole model, a generalization of the exciton-coupling theory applicable to a wider class of scattering systems [33], was applied in Fig. 15 to a chiral dimer of gold NRs separated by a distance of $100 \mathrm{~nm}$; the result- 


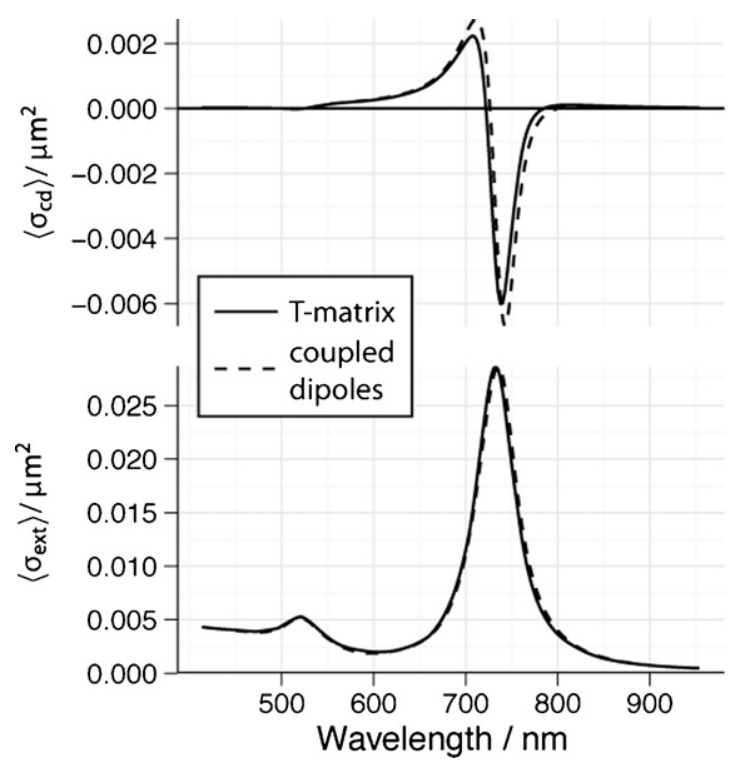

Figure 15 Numerical simulation of the orientation-averaged extinction (bottom) and CD (top) spectra of a chiral dimer of gold nanorods, represented as prolate ellipsoids with semi-axes dimensions $15 \mathrm{~nm} \times 40 \mathrm{~nm}$, with parameters $\varphi=\pi / 4, \alpha_{1}=\alpha_{2}=0$, $d=100 \mathrm{~nm}$. The surrounding medium has a refractive index of 1.5 (glass). The solid curve was obtained using the coupleddipole approximation, with the polarizability prescription of Kuwata et al. [131]. The dashed curve is the rigorous multiplescattering solution of the Maxwell equations for the same geometry including multipole orders up to $l_{\max }=15[132,134]$.

ing extinction (Fig. 15a) and CD spectra (Fig. 15b) are found to be in good agreement with the rigorous solution of the Maxwell equations [132-134]. The CD predicted for these plasmonic structures reveals several notable features. A strong bisignated $C D$ band is predicted in the spectral region of the longitudinal LSPR $(\lambda \sim 720 \mathrm{~nm})$. The transverse LSPR $(\lambda \sim 520 \mathrm{~nm})$, much weaker in extinction, is not visible in the predicted $C D$ spectrum, in support of the simplifying assumption of neglecting the effect of the transverse LSPR. In the bisignated CD lineshape, the antisymmetric (dark) mode, despite having a small electric dipole moment, is observed with the same integrated intensity as its bright counterpart. This interesting prediction originates from the characteristic property of circular dichroism, which arises from the scalar product of the electric dipole moment and the magnetic dipole moment of the structure [28]. We also note that a general sum rule $\int_{0}^{\infty} \sigma_{C D} d \omega=0$ constrains the integrated CD spectrum [46].

As the scale of the system increases from the molecular regime well-described by exciton-coupling theory to larger nanostructures, a rich variety of effects should arise that are unique to the plasmonic case. Scattering of light by the particles, negligible in very small systems, may strongly perturb, and eventually dominate, the optical activity. Additionally, retardation effects in dimers with a separation commensurate with the wavelength of the incident light affect the optical properties and would lead to an asymmetric spectral lineshape [28].

Influence of the particle aspect ratio: Govorov and coworkers recently proposed and theoretically investigated for
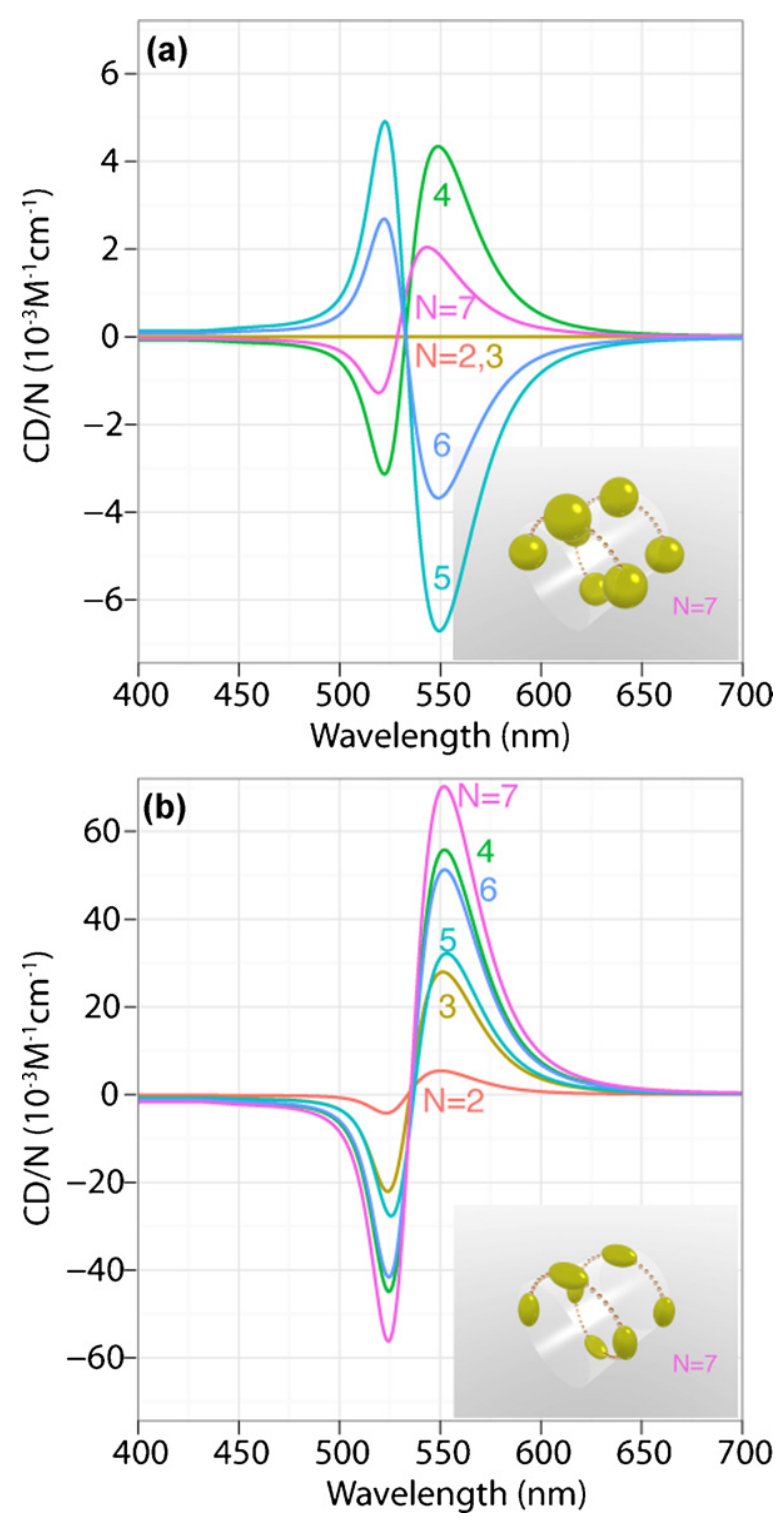

Figure 16 Simulated CD spectra using a coupled-dipole model. Nanoparticles immersed in a homogeneous medium of refractive index 1.33 (water) are arranged following a helical curve with $12 \mathrm{~nm}$ radius and $15 \mathrm{~nm}$ pitch. The nanoparticles are placed every $90^{\circ}$ of gyration on the helix. The simulated spectra are scaled by the number of particles $N(N=2-7)$. (a) Spheres with $5 \mathrm{~nm}$ radius, reproducing the results of Govorov and coworkers [28]. (b) Prolate ellipsoids of semi-axes $4.5 \mathrm{~nm} \times 5 \mathrm{~nm}$ (aspect ratio 1:1.1) with the major axis tangential to the helix. Adapted with permission from Ref. [34]. Copyright @2011 Wiley$\mathrm{VCH}$.

first time the circular dichroism properties of assemblies of gold nanospheres [28]. With a diameter $<10 \mathrm{~nm}$, the spheres studied in this work are well characterized by the dipolar approximation, and their plasmonic response is dominated by absorption. Chiral assemblies in the form of helices were predicted to exhibit circular dichroism, as well as dissymmetric tetramers. However, it was noted that the spectral lineshape of the SP-CD signal would be remarkably sensitive to the precise geometry of the assembly, which can 
be responsible for the experimental observation of relatively weak CD signals in such structures, but undoubtedly of interest for future sensing applications. A variation in the number of particles in the helical structure, or a slight change in the size of one particle in the tetramer could result in a complete sign flip of the SP-CD bands (Fig. 16a). The authors concluded with an original comparison between the relative efficiency of the plasmonic structures and chemical compounds. When normalized by the volume of the nanostructure, the $C D$ strength reached a value below, but of the order of, that for organic molecules with record CD.

A robust SP-CD has been predicted for a chiral structure bearing anisotropic particles that are oriented along a helix [34] (Fig. 16b). This prediction was experimentally confirmed by the intense optical activity observed in gold nanorods organized in 3D chiral nanocomposites (see Section Collective chirality in plasmonic nanoparticles). Notably, a chiral arrangement of nanoparticles requires at least four nanospheres but only two nanorods. In addition, the intensity of the normalized SP-CD rapidly increases with the number of NRs in the assembly. Surprisingly, even a slight departure from sphericity (aspect ratio 1:1.1) is sufficient to greatly improve the robustness of the $C D$ lineshape with respect to the number of the particles present in the system. More generally, it is well known that anisotropic particles such as gold nanorods provide more intense and sensitive plasmonic UV-vis response with respect to gold nanospheres. Indeed, spherical gold particles suffer from a number of limitations in their optical properties such as the lack of tunability, and the relatively low quality factor of their LSPR [135].

Perspectives: These encouraging predictions are a strong call for experimental verification, using the vast bestiary of colloidal metal particles. To mention one possible study, in Fig. 17 a strong dependence of the anisotropy factor with the nanoparticle aspect ratio has been predicted, in the simple configuration depicted in Fig. 14a (a dimer of gold nanorods) [46]. The advantage of using non-spherical particles such as gold nanorods is obvious; the intensity of the SP-CD effect undergoes a large amplification as soon as the particles depart from sphericity. Understanding the effect of particle volume on the $C D$ strength is also an interesting pursuit, as with increasing particle volume the influence of radiative damping plays a more important role. We also suggest a promising and complementary support of purely optical characterization techniques for spatially resolved optical activity measurements, in the form of electron-gain spectroscopy and cathodoluminescence [136]. These two techniques have recently shown great promise in the fine characterization of plasmonic nanostructures, but to the best of our knowledge have not yet been applied to the particular study of chiral nanostructures.

Further studies will probably call for more accurate modeling tools that extend beyond the limitations of the coupled-dipole approach. In particular, multipole expansions are necessary to acknowledge the contribution of higher order modes which are typically encountered in larger particles or short particle-particle distances. In this regard, we point out the T-matrix method as a promising framework, with flexibility in the description of particles of arbitrary shape [137], and, perhaps as importantly, the efficient treat-

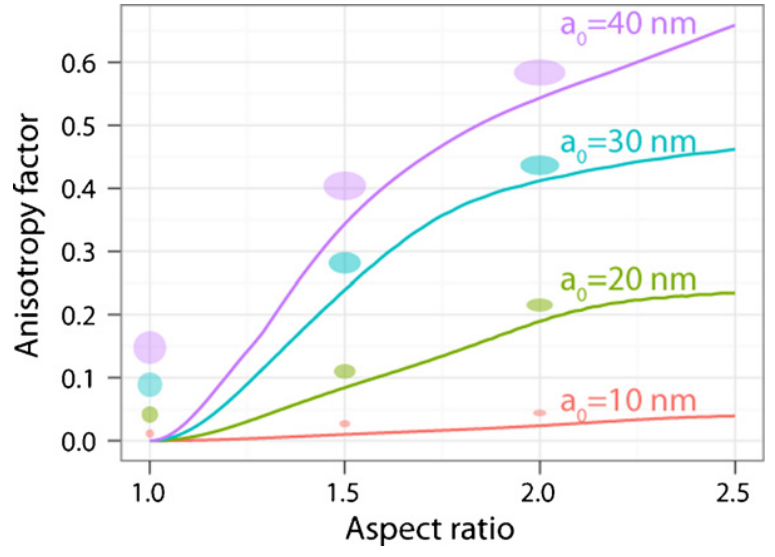

Figure 17 Simulated anisotropy factor g using a coupleddipole model for a chiral dimer of gold nanorods immersed in a homogeneous medium of refractive index 1.5 (typical value for glass). The nanoparticles are separated by a center-tocenter distance $d=100 \mathrm{~nm}$. The dihedral angle is fixed at $\pi / 4$, and $\alpha_{1}=\alpha_{2}=0$ (see Fig. 14). For a fixed volume of the particles, specified as the radius $a_{0}$ of an equi-volume sphere, the aspect ratio is varied from 1:1 (sphere, zero CD signal) to 1:2.5 (prolate ellipsoid). The reported $g$-factor is taken at its maximum value, which occurs at different wavelengths for different aspect ratios.

Adapted from Ref. [46]. Copyright @2011 American Chemical Society.

ment of multiple-scattering [138] and orientation-averaging [139]. Another elegant approach recently proposed by Giessen and co-workers considers the extension of the hybridization picture to more complex structures with strong multipolar and magnetic response, such as coupled split-ring resonators [140]. We conclude by noting that complex nanostructures may not only be produced to present exotic optical activity properties through optimized coupling of meta-atoms, but also in shaping the intrinsic chirality of the exciting field itself $[141,142]$.

\section{Conclusions and outlook}

We intended to introduce in this review novel gold and silver nanostructures with individual or collective chirality, which are prepared via colloidal synthesis methodologies and possess the desirable aspects of enantioselectivity and enantiospecificity characteristics of chiral molecules prepared by synthetic organic chemistry. The use of chiral ligands in the proximity of nanoclusters and nanoparticles has already led to the synthesis of important novel nanomaterials with individual dissymmetry, such as intrinsically chiral nanoparticles [54,55], capped nanocrystals with a chiral distribution of the electron density $[61,75]$, and footprinted nanoparticles $[64,65]$. Although still in their infancy, these systems have shown very high catalytic activity and enantioselectivity toward several organic transformations $[66,143]$, and can be considered potential electrochemical [144], VCD [57], and SEROA (surface enhanced Raman optical activity) [145] sensors. However, the potential uses of nanoparticles with individual chirality as a plasmonic material $[52,76]$ is still hindered primarily due to limitations in 
colloidal synthesis. An approach that might overcome these limitations is the rational design of colloidal heterostructured nanocrystals [146], in which the spatially controlled distribution of the chemical composition and, consequently, of the different reactivities, could be used to induce the anisotropic growth of metallic branches, different metal nanoparticle junctions, and/or local twists in controlled directions (see Fig. 9a).

Moreover, the systematic integration of nanotechnology with well-established, traditional areas of study such as polymer and supramolecular chemistry has, at a relatively early stage, played a key role in the development of useful collective chiral nanocomposites with unique plasmonic properties [34-36,41]. Following the seminal article by Pendry [18], the pursuit of negative refraction has seen an intense activity in the design of chiral metamaterials [19]. Experimental works were first realised at microwave frequencies [147], and have recently been scaled down to the visible [148]. If a negative index of refraction is to be achieved with this route, it should require an intense optical activity, good transparency, and a homogeneous composition on the scale of the working wavelength. While a variety of designs have been proposed $[19,148]$, the focus has been largely on periodic, often 2D structures. Such structures are often limited to work at fixed incidence and the freedom of design is constrained by the current limitations of lithographic techniques. In contrast, colloidal synthesis offers the perspective of achieving truly $3 \mathrm{D}$ chiral structures, by a versatile and complementary (chemical) manufacturing process. More than offering a mere alternative to conventional arrays of plasmonic resonators (typically split ring resonators $[121,140]$, crosses [149], or gammadions [20]), we expect that plasmonic nanostructures obtained by colloid chemistry methods will enrich the emerging field of metamaterials and widen its spread of applications. The coming years will surely witness the development of hybrid systems that fall somewhere between the current boundary of periodic metamaterials fabricated by lithographic techniques, and the colloidal synthesis route.

Finally, and taking into account the high intensity of anisotropy factors that have been registered by plasmonic nanoparticles in the visible-near infrared region [34-36,41], using versatile and generic self-assembly strategies, we anticipate the use of such plasmonic nanoantennas as powerful chirality probes upon attachment to proteins and DNA for in situ structure determination $[20,150]$ by selecting the appropriate nature, dimensions, morphology and functionalization of the metal nanoparticles. With a solution-based chiral metamaterial, the external chiral template may be biological (DNA, bacteria, viruses, ...), thereby offering a wide range of possible applications beyond the realm of stereochemistry in life.

\section{Acknowledgements}

The authors would like to thank Javier García de Abajo (CSIC, Spain) for providing the numerical tools used in the T-matrix calculation of Fig. 15. LMLM acknowledges funding from the Spanish Ministerio de Ciencia e Innovación (MAT2010-15374), Xunta de Galicia (09TMT011314PR), and the EU (INGENIOUS, Grant no. CP-248236; NANODIRECT, Grant no. CP-FP 213948-
2). JLAG acknowledges funding from the Spanish Ministerio de Ciencia e Innovación (CTQ2010-18576). AGM and JLAG acknowledge the Juan de la Cierva Program (MICINN, Spain) and the Isidro Parga Pondal Program (Xunta de Galicia, Spain), respectively.

\section{References}

[1] G.H. Wagnière, On Chirality and the Universal Asymmetry , VHCA-Wiley-VCH, Zürich, Switzerland, 2007.

[2] M.L. Pasteur, Ann. Chim. Phys. 24 (1848) 442.

[3] Y. Okamoto, T. Ikai, Chem. Soc. Rev. 37 (2008) 2593.

[4] T.P. Yoon, E.N. Jacobsen, Science 299 (2003) 1691.

[5] I. Agranat, H. Caner, A. Caldwell, Nat. Rev. Drug Discov. 1 (2002) 753.

[6] H. Buschmann, R. Thede, D. Heller, Angew. Chem. Int. Ed. 39 (2000) 4033.

[7] J.D. Watson, F.H.C. Crick, Nature 171 (1953) 737.

[8] J.D. Woodward, R. Wepf, B.T. Sewell, J. Microsc. 234 (2009) 287.

[9] P. Vukusic, Science 325 (2009) 398.

[10] G.M. Whitesides, B. Grzybowski, Science 295 (2002) 2418.

[11] G.M. Whitesides, Small 1 (2005) 172.

[12] S. Che, Z. Liu, T. Ohsuna, K. Sakamoto, O. Terasaki, T. Tatsumi, Nature 429 (2004) 281.

[13] A. Sánchez-Castillo, C.E. Román-Velázquez, C. Noguez, L. Meza-Montes, Phys. Rev. B 73 (2006) 045401.

[14] M.P. Moloney, Y.K. Gun'ko, J.M. Kelly, Chem. Commun. (2007) 3900.

[15] C. Gautier, T. Bürgi, ChemPhysChem 10 (2009) 483.

[16] Y. Liu, X. Zhang, Chem. Soc. Rev. 40 (2011) 2494.

[17] U. Gubler, C. Bosshard, Nat. Mater. 1 (2002) 209.

[18] J.B. Pendry, Science 306 (2004) 1353.

[19] B. Wang, J. Zhou, T. Koschny, M. Kafesaki, C.M. Soukoulis, J. Opt. A 11 (2009) 114003.

[20] E. Hendry, T. Carpy, J. Johnston, M. Popland, R.V. Mikhaylovskiy, A.J. Lapthorn, et al., Nat. Nanotechnol. 5 (2010) 783.

[21] M. Faraday, Philos. Trans. R. Soc. London 147 (1857) 145.

[22] G. Mie, Ann. Phys. 330 (1908) 377.

[23] H. Hovel, S. Fritz, A. Hilger, U. Kreibig, M. Vollmer, Phys. Rev. B 48 (1993) 18178.

[24] Y. Xia, Y. Xiong, B. Lim, S.E. Skrabalak, Angew. Chem. Int. Ed. 48 (2009) 60.

[25] R. Shenhar, V.M. Rotello, Acc. Chem. Res. 36 (2003) 549.

[26] R. Alvarez-Puebla, L.M. Liz-Marzán, F.J. García de Abajo, J. Phys. Chem. Lett. 1 (2010) 2428.

[27] C. Noguez, I.L. Garzón, Chem. Soc. Rev. 38 (2009) 757.

[28] Z. Fan, A.O. Govorov, Nano Lett. 10 (2010) 2580.

[29] Lord Kelvin (W.H. Thomson) Baltimore Lectures , C.J. Clay and Sons, London, 1904, p. 619.

[30] A. Guijarro, M. Yus, The Origin of Chirality in the Molecules of Life: a Revision from Awareness to the Current Theories and Perspectives of this Unsolved Problem , Royal Society of Chemistry, 2009.

[31] L.D. Barron, Molecular Light Scattering and Optical Activity , Cambridge University Press, 2004.

[32] N. Berova, D. Bari, G. Pescitelli, Chem. Soc. Rev. 36 (2007) 914.

[33] N. Berova, K. Nakanishi, R.W. Woody, Circular Dichroism: Principles and Applications, Wiley, 2000.

[34] A. Guerrero-Martínez, B. Auguié, J.L. Alonso-Gómez, Z. Džolić, S. Gómez-Graña, M. Žinić, et al., Angew. Chem. Int. Ed. 50 (2011) 5499.

[35] G. Shemer, O. Krichevski, G. Markovich, T. Molotsky, I. Lubitz, A.B. Kotlyar, J. Am. Chem. Soc. 128 (2006) 11006. 
[36] J. George, K.G. Thomas, J. Am. Chem. Soc. 132 (2010) 2502.

[37] W. Chen, A. Bian, A. Agarwal, L. Liu, H. Shen, L. Wang, et al., Nano Lett. 9 (2009) 2153.

[38] I. Lieberman, G. Shemer, T. Fried, E.M. Kosower, G. Markovich, Angew. Chem. Int. Ed. 47 (2008) 4855.

[39] T. Molotsky, T. Tamarin, A.B. Moshe, G. Markovich, A.B. Kotlyar, J. Phys. Chem. C114 (2010) 15951.

[40] Y. Li, M. Liu, Chem. Commun. (2008) 5571.

[41] H.S. Oh, S. Liu, H. Jee, A. Baev, M.T. Swihart, P.N. Prasad, J. Am. Chem. Soc. 132 (2010) 17346.

[42] R.S. Walters, C.M. Kraml, N. Byrne, D.M. Ho, Q. Qin, F.J. Coughlin, et al., J. Am. Chem. Soc. 130 (2008) 16435.

[43] J.L. Alonso-Gómez, P. Rivera-Fuentes, N. Harada, N. Berova, F. Diederich, Angew. Chem. Int. Ed. 48 (2009) 5545.

[44] P. Rivera-Fuentes, J.L. Alonso-Gómez, A.G. Petrovic, F. Santoro, N. Harada, N. Berova, et al., Angew. Chem. Int. Ed. 49 (2010) 2247.

[45] R.M. Pearlstein, R.C. Davis, S.L. Ditson, Proc. Natl. Acad. Sci. U.S.A. 79 (1982) 400.

[46] B. Auguié, J.L. Alonso-Gomez, A. Guerrero-Martínez, L.M. LizMarzán, J. Phys. Chem. Lett. 2 (2011) 846.

[47] A.G. Petrovic, A. Navarro-Vázquez, J.L. Alonso-Gómez, Curr. Org. Chem. 14 (2010) 1612.

[48] R. Jin, Nanoscale 2 (2010) 343.

[49] B. Wiley, Y. Sun, Y. Xia, Acc. Chem. Res. 40 (2007) 1067.

[50] G.S. Meǐtraux, C.A. Mirkin, Adv. Mater. 17 (2005) 412.

[51] J. Ha, A. Solovyov, A. Katz, Langmuir 25 (2009) 153.

[52] A.O. Govorov, Z. Fan, P. Hernandez, J.M. Slocik, R.R. Naik, Nano Lett. 10 (2010) 1374.

[53] N. Cathcart, V. Kitaev, J. Phys. Chem. C 114 (2010) 16010.

[54] N. Cathcart, P. Mistry, C. Makra, B. Pietrobon, N. Coombs, M. Jelokhani-Niaraki, et al., Langmuir 25 (2009) 5840.

[55] N. Nishida, H. Yao, T. Ueda, A. Sasaki, K. Kimura, Chem. Mater. 19 (2007) 2831.

[56] H. Yao, M. Saeki, K. Kimura, J. Phys. Chem. C 114 (2010) 15909.

[57] M. Bieri, C. Gautier, T. Bürgi, Phys. Chem. Chem. Phys. 9 (2007) 671.

[58] J.F. Parker, C.A. Fields-Zinna, R.W. Murray, Acc. Chem. Res. $43(2010) 1289$.

[59] T.G. Schaaff, G. Knight, M.N. Shafigullin, R.F. Borkman, R.L. Whetten, J. Phys. Chem. B 102 (1998) 10643.

[60] T.G. Schaaff, R.L. Whetten, J. Phys. Chem. B 104 (2000) 2630.

[61] M.R. Provorse, C.M. Aikens, J. Am. Chem. Soc. 132 (2010) 1302.

[62] I.L. Garzón, J.A. Reyes-Nava, J.I. Rodríguez-Hernández, I. Sigal, M.R. Beltrán, K. Michaelian, Phys. Rev. B 66 (2002) 734031.

[63] I.E. Santizo, F. Hidalgo, L.A. Pérez, C. Noguez, I.L. Garzón, J. Phys. Chem. C 112 (2008) 17533.

[64] Y. Negishi, K. Nobusada, T. Tsukuda, J. Am. Chem. Soc. 127 (2005) 5261.

[65] S. Si, C. Gautier, J. Boudon, R. Taras, S. Gladiali, T. Bürgi, J. Phys. Chem. C 113 (2009) 12966.

[66] E.S. Andreiadis, M.R. Vitale, N. Mézailles, X. Le Goff, P. Le Floch, P.Y. Toullec, et al., Dalton Trans. 39 (2010) 10608.

[67] M.W. Heaven, A. Dass, P.S. White, K.M. Holt, R.W. Murray, J. Am. Chem. Soc. 130 (2008) 3754.

[68] Z. Wu, C. Gayathri, R.R. Gil, R. Jin, J. Am. Chem. Soc. 131 (2009) 6535.

[69] A. Sánchez-Castillo, C. Noguez, I.L. Garzón, J. Am. Chem. Soc. 132 (2010) 1504.

[70] S.K. Basiruddin, A. Saha, N. Pradhan, N.R. Jana, J. Phys. Chem. C 114 (2010) 11009.

[71] K.G. Thomas, P.V. Kamat, Acc. Chem. Res. 36 (2003) 888.

[72] R.A. Alvarez-Puebla, L.M. Liz-Marzán, Small 6 (2010) 610.

[73] C. Gautier, T. Bürgi, J. Phys. Chem. C 114 (2010) 15897.

[74] C. Gautier, T. Bürgi, J. Am. Chem. Soc. 128 (2006) 11079.
[75] J.M. Slocik, A.O. Govorov, R.R. Naik, Nano Lett. 11 (2011) 701.

[76] I. Carmeli, I. Lieberman, L. Kraversky, Z. Fan, A.O. Govorov, G. Markovich, et al., Nano Lett. 10 (2010) 2069.

[77] K.L. Kelly, E. Coronado, L.L. Zhao, G.C. Schatz, J. Phys. Chem. B 107 (2003) 668.

[78] V.P. Drachev, W.D. Bragg, V.A. Podolskiy, V.P. Safonov, W.-T. Kim, Z.C. Ying, et al., J. Opt. Soc. Am. B 18 (2001) 1896.

[79] B.L. Feringa, R.A. Van Delden, Angew. Chem. Int. Ed. 38 (1999) 3418.

[80] Y.K. Pang, J.C.W. Lee, H.F. Lee, W.Y. Tam, C.T. Chan, P. Sheng, Opt. Express 13 (2005) 7615.

[81] J.K. Gansell, M. Thiel, M.S. Rill, M. Decker, K. Bade, V. Saile, et al., Science 325 (2009) 1513.

[82] T.K. Sau, A.L. Rogach, Adv. Mater. 22 (2010) 1781.

[83] J. Pérez-Juste, I. Pastoriza-Santos, L.M. Liz-Marzán, P. Mulvaney, Coord. Chem. Rev. 249 (2005) 1870.

[84] Y.N. Xia, P.D. Yang, Adv. Mater. 15 (2003) 351.

[85] A. Guerrero-Martínez, S. Barbosa, I. Pastoriza-Santos, L.M. Liz-Marzán, Curr. Opin. Colloid Interface Sci. 16 (2011) 118.

[86] E. Carbó-Argibay, B. Rodríguez-González, S. Gómez-Graña, A. Guerrero-Martínez, I. Pastoriza-Santos, J. Pérez-Juste, et al., Angew. Chem. Int. Ed 49 (2010) 9397.

[87] A. Sánchez-Iglesias, M. Grzelczak, B. Rodríguez-González, R. Alvarez-Puebla, L.M. Liz-Marzán, N.A. Kotov, Langmuir 25 (2009) 11431.

[88] M. Grzelczak, J. Vermant, E.M. Furst, L.M. Liz-Marzán, ACS Nano 4 (2010) 4.

[89] A.J. Mastroianni, S.A. Claridge, A.P. Alivisatos, J. Am. Chem. Soc. 131 (2009) 8455.

[90] M. Yurkin, A. Hoekstra, J. Quant. Spectrosc. Radiat. Transfer 106 (2007) 558.

[91] F.J. García de Abajo, Rev. Mod. Phys. 79 (2007) 1267.

[92] W.D. Bragg, V.P. Safonov, W. Kim, K. Banerjee, M.R. Young, J.G. Zhu, et al., J. Microsc. 194 (1999) 574.

[93] S.T. Selvan, T.T.Y. Tan, D.K. Yi, N.R. Jana, Langmuir 26 (2010) 11631.

[94] J.M. Romo-Herrera, R.A. Alvarez-Puebla, L.M. Liz-Marzán, Nanoscale 3 (2011) 1304.

[95] M.-C. Daniel, D. Astruc, Chem. Rev. 104 (2004) 293.

[96] A.P. Alivisatos, K. Johnsson, X. Peng, T. Wilson, C. Loweth, M. Bruchez Jr., et al., Nature 382 (1996) 609.

[97] X. Yang, L.A. Wenzler, J. Qi, X. Li, N.C. Seeman, J. Am. Chem. Soc. 120 (1998) 9779.

[98] C.M. Erben, R.P. Goodman, A.J. Tuberfield, J. Am. Chem. Soc. 129 (2007) 6992.

[99] W.M. Shih, J.D. Quispe, G.F. Joyce, Nature 427 (2004) 618.

[100] R. Goodman, I.A.T. Schaap, C.F. Tardin, C.M. Erben, R.M. Berry, C.F. Schmidt, et al., Science 310 (2005) 1661.

[101] H. Jaganathan, J.M. Kinsella, A. Ivanisevic, ChemPhysChem 9 (2008) 2203.

[102] S. Roy, S. Basak, A.K. Dasgupta, J. Nanosci. Nanotechnol. 10 (2010) 819.

[103] T. Li, H.G. Park, H.-S. Lee, S.-H. Choi, Nanotechnology 15 (2004) $\mathrm{S660.}$

[104] F. Leroux, M. Gysemans, S. Bals, K.J. Batenburg, J. Snauwaert, T. Verbiest, et al., Adv. Mater. 22 (2010) 2193.

[105] Linear dichroism (LD) effects cannot be excluded from the $C D$ measurements in these experimental conditions due to the strong macroscopic anisotropy characteristics of gels or films.

[106] F. Hidalgo, A. Sánchez-Castillo, C. Noguez, Phys. Rev. B 79 (2009) 075438.

[107] S.A. Maier, Plasmonics: Fundamentals and Applications , Springer, New York, USA, 2007.

[108] B.T. Draine, P.J. Flatau, J. Opt. Soc. Am. A 11 (1994) 1491.

[109] M.A. Yurkin, A.G. Hoekstra, J. Quant. Spectrosc. Radiat. Transfer 106 (2007) 558. 
[110] B.T. Draine, Astrophys. J. 333 (1988) 848.

[111] C.E. Roman-Velazquez, C. Noguez, I.L. Garzón, J. Phys. Chem. B 107 (2003) 12035, We are grateful to the authors for providing cluster atomic coordinates.

[112] I. Garzón, M. Beltrán, G. González, I. Gutiérrez-González, K. Michaleian, J. Reyes-Nava, et al., Eur. Phys. J. D 24 (2003) 105.

[113] Z.-Y. Zhang, Y.-P. Zhao, Appl. Phys. Lett. 90 (2007) 221501.

[114] G.C. Schatz, L. Jensen, K.K. Kelly, A.A. Lazarides, J. Clust. Sci. 10 (1999) 295.

[115] W. Kuhn, Trans. Far. Soc. 26 (1930) 293.

[116] M. Born, Proc. R. Soc. A 150 (1935) 84.

[117] P.K. Jain, S. Eustis, M.A. El-Sayed, J. Phys. Chem. B 110 (2006) 18243.

[118] N. Harada, Y. Takuma, H. Uda, J. Am. Chem. Soc. 100 (1978) 4029.

[119] N. Harada, Circular Dichroic Spectroscopy; Exciton Coupling in Organic Stereochemistry , University Science Books, Mill Valley, USA, 1983.

[120] P. Nordlander, C. Oubre, E. Prodan, K. Li, M.I. Stockman, Nano Lett. 4 (2004) 899.

[121] N.B. Liu, H.A. Giessen, Angew. Chem. Int. Ed. 49 (2010) 9838.

[122] T.J. Davis, D.E. Gómez, K.C. Vernon, Nano Lett. 10 (2010) 2618.

[123] M. Schmeits, L. Dambly, Phys. Rev. B 44 (1975) 12706.

[124] E. Prodan, C. Radloff, N.J. Halas, P. Nordlander, Science 302 (2003) 419.

[125] P.K. Jain, W. Huang, M.A. El-Sayed, Nano Lett. 7 (2007) 2080.

[126] V. Myroshnychenko, J. Rodriguez-Fernandez, I. PastorizaSantos, A.M. Funston, C. Novo, P. Mulvaney, et al., Chem. Soc. Rev. 37 (2008) 1792.

[127] P.K. Jain, M.A. El-Sayed, Chem. Phys. Lett. 487 (2010) 153.

[128] P. Pramod, K.G. Thomas, Adv. Mater. 20 (2008) 4300.

[129] B. Willingham, D.W. Brandl, P. Nordlander, Appl. Phys. B 93 (2008) 209.

[130] A. Funston, C. Novo, T. Davis, P. Mulvaney, Nano Lett. 9 (2009) 1651.

[131] H. Kuwata, H. Tamaru, K. Esumi, K. Miyano, Appl. Phys. Lett. 83 (2003) 4625.

[132] F.J. García de Abajo, A. Howie, Phys. Rev. Lett. 80 (1998) 5180.

[133] F.J. García de Abajo, Phys. Rev. B 60 (1999) 6086.

[134] F.J. García de Abajo, A. Howie, Phys. Rev. Lett. 65 (2002) 115418.

[135] C. Sönnichsen, T. Franzl, T. Wilk, G. von Plessen, J. Feldmann, O. Wilson, et al., Phys. Rev. Lett. 88 (2002) 077402.

[136] F.J. García de Abajo, Rev. Mod. Phys. 82 (2010) 209.

[137] J. Hellmers, T. Wriedt, J. Quant. Spectrosc. Radiat. Transfer 106 (2007) 90.

[138] M.I. Mishchenko, L. Travis, A.A. Lacis, Scattering, Absorption, and Emission of Light by Small Particles , Cambridge University Press, Cambridge IK, 2002.

[139] M.I. Mishchenko, Astrophys. Space Sci. 164 (1990) 1.

[140] N. Liu, H. Liu, S. Zhu, H. Giessen, Nat. Photon. 3 (2009) 157.

[141] Y. Tang, A.E. Cohen, Phys. Rev. Lett. 104 (2010) 1.

[142] N. Gadegaard, M. Kadodwala, Nat. Nanotechnol. 5 (2010) 783.

[143] P. Barbaro, V.D. Santo, F. Liguori, Dalton Trans. 39 (2010) 8391.

[144] Y.-J. Kang, J.-W. Oh, Y.-R. Kim, J.S. Kim, H. Kim, Chem. Commun. 46 (2010) 5665.

[145] R. Lombardini, R. Acevedo, N.J. Halas, B.R. Johnson, J. Phys. Chem. C 114 (2010) 7390.

[146] L. Carbone, P.D. Cozzoli, NanoToday 5 (2010) 449.

[147] V.M. Shalaev, Nat. Photon. 1 (2007) 41.

[148] S. Zhang, Y.-S. Park, J. Li, X. Lu, W. Zhang, X. Zhang, Phys. Rev. Lett. 102 (2009) 023901.

[149] J. Zhou, J. Dong, B. Wang, T. Koschny, M. Kafesaki, C.M. Soukoulis, Phys. Rev. B 79 (2009) 121104.

[150] M.J. García-Parajo, Nat. Photon. 2 (2008) 201.

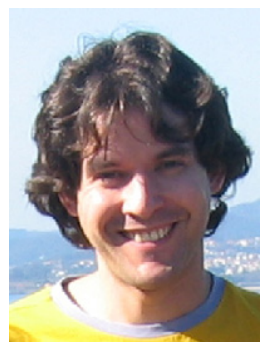

Andrés Guerrero-Martínez received his $\mathrm{PhD}$ degree in Chemistry under the supervision of Prof. G. Tardajos at the University Complutense in 2006 (Madrid, Spain), where he served as lecturer of Physical Chemistry between 2006 and 2007. He then joined the group of Prof. L. De Cola at the University of Münster (Germany), as a postdoctoral fellow from 2007 to 2009. Since 2009, he holds a Juan de la Cierva Postdoctoral Fellowship in the group of Prof. L. M. Liz-Marzán at the University of Vigo (Spain), where he is investigating nanostructured materials with tailored optical properties.

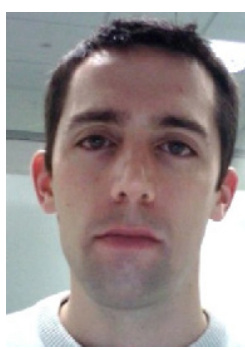

Baptiste Auguié studied physics in Rennes, France, and in Montréal, Canada. Working on the optical properties of gold nanostructures, he obtained his PhD in 2009 from the University of Exeter, UK, under the supervision of Prof. W.L. Barnes. He spent the following year as a post-doctoral research fellow with Prof. J.G. de Abajo (CSIC, Madrid), and Prof. L. LizMarzán (Vigo, Spain). He is currently working on Surface-Enhanced Raman scattering in the group of Prof. P. Etchegoin (Wellington, New

Zealand).

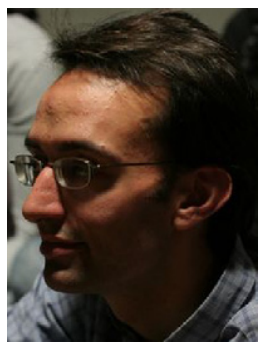

José Lorenzo Alonso Gómez obtained his $\mathrm{PhD}$ degree in 2006 from the University of Vigo. Shortly after, he moved to ETH Zurich and then to Columbia University for his postdoctoral research at the groups of Prof. F. Diederich and Prof. N. Berova respectively. During this period he got involved in the synthesis of chiral macromolecules as well as in the study of the mechanisms origin of their chiroptical responses. Back to Spain, he obtained a research position at the University of Vigo. At the moment he is the principal investigator of a project on the study of the chiral induction and chiral amplification via interaction of enantiopure allenophanes and metal nanoparticles.

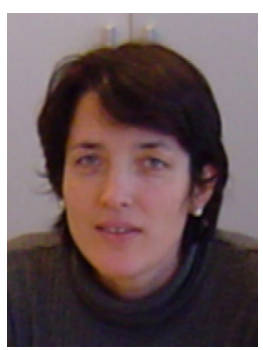

Magdalena Cid did her PhD at the Universidade of Santiago de Compostela and postdoctoral stages in Novartis (Basel, Switzerland) and The Scripps Research Institute (La Jolla, CA, USA) mainly dealing with synthesis of nitrogen-containing compounds. In 1996 she joined the Universidade de Vigo where she is an associate professor since 1999. She is interested in the design, synthesis and characterization of chiral (macro)molecules and the usage of chiroptical properties and NMR (scalar and dipolar coupling constants) for total elucidation of their structures.

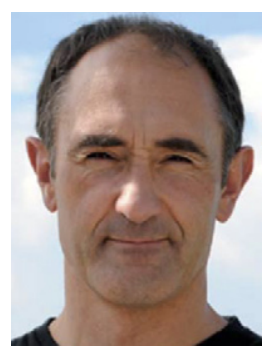

Luis M. Liz-Marzán received his PhD from the University of Santiago de Compostela in 1992. He worked as a postdoctoral fellow at the Van't Hoff Laboratory (Utrecht University) and (more recently) as visiting professor at Tohoku University, the University of Michigan, University of Melbourne and University of Hamburg. He joined the University of Vigo in 1995, where he currently is a Full Professor and leader of the Colloid Chemistry Group. He is recipient of several research awards, editor and editorial advisory board member of several chemistry, nanotechnology and materials science journals. His current interests include the synthesis and assembly of nanoparticles, nanoplasmonics, and the development of nanoparticle-based biosensors. 